\title{
Estructura y COMPOSición florística del CerRado en el Parque Nacional Noel Kempff Mercado, Santa Cruz, Bolivia
}

\author{
Bonifacio Mostacedo C.* y Thimoty J. Killeen** \\ *Museo de Historia Natural Noel Kempff Mercado, Universidad Autónoma Gabriel René Moreno, \\ Casilla Postal 2489, Santa Cruz de la Sierra, Bolivia. \\ ${ }^{* *}$ Missouri Botanical Garden, P. O. Box 299, St. Louis, Missouri 63166-0299, USA.
}

\begin{abstract}
Resumen. Se estudió la estructura y composición florística del Cerrado en dos sitios del Parque Nacional "Noel Kempff Mercado", Santa Cruz, Bolivia. Los resultados indican que los sitios están ubicados en dos comunidades diferentes de Cerrado; el primero corresponde al campo cerrado, y el segundo al cerradâo El estrato inferior a $50 \mathrm{~cm}$ está dominado por graminoides en ambos sitios, mientras que los estratos superiores están dominados por subarbustos y arbustos en el campo cerrado, y árboles en el cerradâo. Se encontraron 41 familias, 90 géneros y 112 especies en el sitio del campo cerrado, y 37 familias, 89 géneros y 106 especies, en el cerradâo. Las Gramineae (29 especies, $51 \%$ ), Leguminosae (23 especies, 4.8-5.7\%) y Guttiferae ( 3 especies, $1-20 \%$ ) son las familias de mayor riqueza florística y/o cobertura. Otras familias importantes por su riqueza florística son Myrtaceae ( 7 y 6 especies), Rubiaceae ( 4 y 6 especies), Euphorbiaceae ( 8 y 2 especies) y Melastomataceae ( 2 y 6 especies) en campo cerrado y cerradâo, respectivamente. En el campo cerrado, las hierbas representan la mayor riqueza de especies, seguidas por graminoides y arbustos; en el cerradâo, los arbustos, hierbas y graminoides representan el mayor número de especies. La cobertura de graminoides y árboles es el más alto en los dos sitios, aunque la cobertura de árboles es casi el doble en el cerradâo. El índice de Sørensen obtenido, nos indica que existe sólo el 39\% de similitud florística entre los dos sitios de
\end{abstract} estudio.

Palabras clave: estructura, florística, Cerrado, Bolivia

Abstract. We studied the structure and floristic composition of the Cerrado vegetation in two sites at the Parque Nacional "Noel Kempff Mercado", Santa Cruz, Bolivia. The results indicated that the sites are located within two different types of Cerrado communities: 1) cerrado and, 2) cerradâo. The lower stratum (50 $\mathrm{cm}$ ) was dominated by grasses, while higher strata were dominated by shrubs and sub-shrubs in the cerrado, and by trees in the cerradâo. We founded 41 families, 90 genera, and 112 species in the cerrado, and 37 families, 39 genera and 106 species in the cerradâo. For both sites, the plant families with greater coverage and species richness were: Gramineae (29 species, $51 \%$ cover), Leguminosae (23 species, $4.8-5.7 \%$ cover) and Guttiferae (3 species, 1-20\% cover). Other important families were: Myrtaceae, Rubiaceae, Euphorbiaceae and Melastomataceae, with 7,6 species; 4,6 species; 8, 2 species; 2, 6 species for cerrado and cerradâo, respectively. Herbs are the most important for the number of species, followed by grasses and shrubs in the cerrado and by shrubs, followed by herbs and grasses in the cerradâ. Floristic similarity between the two communities was relatively low (39\% according to Sørensen index).

Key words: Structure, floristics, Cerrado, Bolivia

$\mathbf{L}$ as sabanas neotropicales ocupan más de dos millones de $\mathrm{km}^{2}$, en su mayor parte situadas en Brasil (Cerrado), Colombia y Venezuela (llanos; Sarmiento, 1983), y el conocimiento sobre su funcionamiento y manejo ha mejorado notablemente en las últimas décadas (Di Stéfano et al., 1995). Sin embargo, Bolivia es uno de los países de Sudamérica con formaciones biogeográficas muy diversas que carece de información básica sobre los recursos naturales renovables (Navarro, 1994). En Bolivia convergen cinco regiones fitogeográficas importantes: la Andina, la Amazónica, el Cerrado, el Chaqueño, y el Complejo del Pantanal (Moraes y Beck, 1992; Beck et al., 1995). Cada región puede ser clasificada en distintas formaciones tomando criterios de latitud, altitud y geomorfología (Beck et al., 1995). Una de las regiones 
fitogeográficas más importantes y que está mejor representada en el departamento de Santa Cruz, es el Cerrado, que se extiende principalmente al norte y este en el Escudo Precámbrico en las provincias Ñuflo de Chávez, Chiquitos, Guarayos, y Velasco (Navarro, 1992; Beck et al., 1993).

El Cerrado (sensu lato) es un término utilizado por los brasileños que significa "denso" y se refiere a una serie de comunidades vegetales de sabanas (Eiten, 1972; Sarmiento, 1983). Estas comunidades se pueden distinguir por la cobertura y altura de los árboles, e incluyen sabanas abiertas dominadas principalmente por gramíneas (campo limpo, campo sujo, campo rupestre), matorrales con árboles de 2-8 m de altura y de $10-30 \%$ de cobertura (campo cerrado y cerrado), y bosques bajos con árboles de 8-18 m de altura y cobertura mayor a $30 \%$ (cerradâo; Goodland, 1971; Coutinho, 1978a; Eiten, 1990). En Bolivia, existen términos que reflejan esta variabilidad, y son comparables a la terminología brasileña como pampa, pampa arbolada, y arbolera (Killeen et al., 1990).
En el Parque Nacional "Noel Kempff Mercado (PNK) del departamento de Santa Cruz se conserva un ejemplo muy representativo del Cerrado, que necesita ser estudiado desde el punto de vista de su flora y ecología, para tener mejor criterio de planificación con fines de conservación y manejo (Killeen et al., 1993; Beck et al., 1995).

El objetivo de este estudio fue analizar la estructura y composición florística de la formación vegetal del Cerrado en dos sitios diferentes del Parque.

\section{Área de estudio}

El estudio se realizó en el PNK, ubicado en el sector nordeste de la provincia Velasco del departamento de Santa Cruz (figura 1). El Parque tiene una superficie de aproximadamente 750,000 ha; geográficamente está ubicado entre las coordenadas: $13^{\circ} 30^{\prime}$ y $14^{\circ} 55^{\prime}$, Latitud S, y $60^{\circ} 20^{\prime}$ y $61^{\circ} 10^{\prime}$ Longitud $O$, con altitudes que varían entre 200 y 300 m s.n.m. (Killeen, no publ.). Las áreas de estudio están situadas en la zona

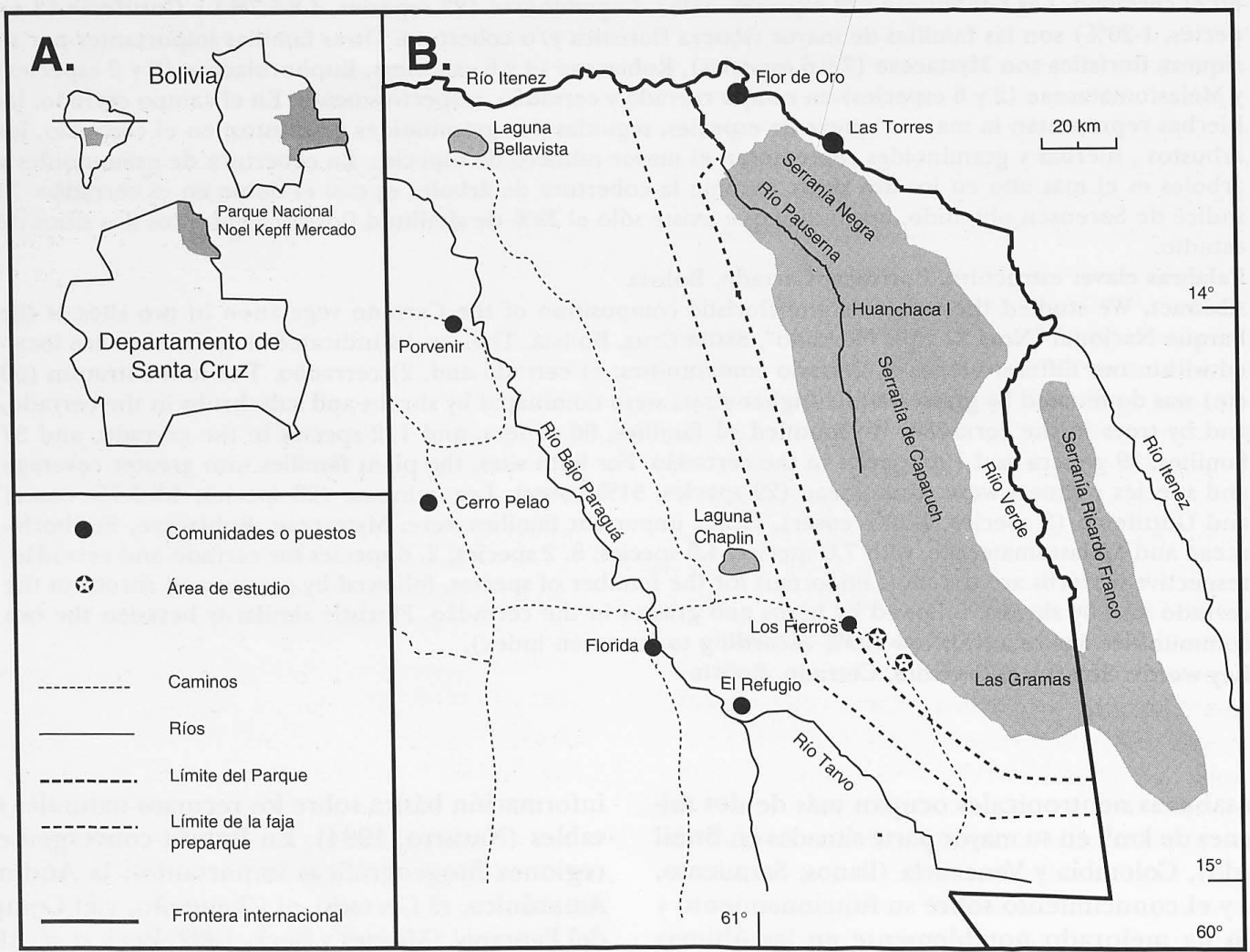

Figura 1. Ubicación geográfica de la zona de estudio. a) Mapa del departamento de Santa Cruz en Bolivia y Sur América. b) Detalle del Parque Nacional "Noel Kempff Mercado", indicando los sitios de estudio. 
Sur del Parque, próximos a las poblaciones de Florida y el campamento Los Fierros que fue utilizado como el centro de operaciones.

Clima. El Parque no cuenta con una estación meteorológica con datos confiables, pero un mapa de isoyetas e isotermas del departamento de Santa Cruz indican que esta zona tiene $1500 \mathrm{~mm} /$ año de precipitación y $25^{\circ} \mathrm{C}$ de temperatura (Cordecruz, 1992; Roche y Rocha, 1985); probablemente los promedios reales están alrededor de estos valores. No obstante, en algunos sitios del Parque, por efectos microclimáticos debido a la presencia de la serranía, pueden registrarse valores mayores de precipitación a los estimados.

Vegetación. Los inventarios preliminares e imágenes de satélite indican que el PNK tiene un mosaico de bosques, sabanas, tierras húmedas y afloramientos de rocas. El bosque tropical húmedo está presente como una franja al pie de la Serranía de Caparuch y en el Valle del Río Verde; al oeste la Reserva Forestal "Bajo Paraguá" está cubierta por la misma formación boscosa. Los bosques semideciduos y secos existen como manchones sobre suelos pedregosos en las faldas de la serranía y alrededor de los afloramientos graníticos; esta vegetación predominante se encuentra al sudoeste del Parque en la zona del Alto Paraguá. (Killeen, no publ.). Los bosques de galería ocupan una franja de 50 a $200 \mathrm{~m}$ de ancho en el fondo del valle de la meseta, a lo largo de quebradas y pequeños ríos; datos preliminares indican que son similares a los bosques húmedos del pie de monte (Arroyo, 1995). La formación vegetal del Cerrado está ubicada por encima de la meseta y laderas de los valles que cruzan la Serranía de Caparuch; además, se le encuentra en las planicies aluviales del Alto Paraguá formando una franja alrededor de las sabanas inundadas (Ruíz y Saravia, 1989; Killeen, no publ.). Las sabanas inundadas están situadas en las planicies aluviales del Alto Paraguá, al sur de la Serranía de Caparuch y al norte en la Estancia Flor de Oro. La diversidad de especies de las sabanas húmedas es grande, debido a la proximidad de diferentes microhábitats con distintos regímenes acuáticos (Beck, 1984; Haase y Beck, 1989). Las lajas son afloramientos de rocas cuarcitas y areniscas ubicadas en la cima de la Serranía de Huanchaca, mientras que los afloramientos graníticos están situados en la planicie al Oeste del Río Paraguá (Killeen et al., 1990).

\section{Métodos}

La investigación para conocer la composición florística y estructura de la vegetación del Cerrado de una región del Parque se realizó en dos sitios. El primer sitio está ubicado a $10 \mathrm{~km}$ al sureste del campamento Los Fierros y a $0.5 \mathrm{~km}$ hacia el este, sobre un camino que se aparta de la carretera principal con rumbo hacia la serranía de Caparuch (14³6'16" S; $60^{\circ} 51^{\prime} 34^{\prime \prime}$ W; 190 m s.n.m.). El segundo sitio está situado sobre el mismo camino hacia la Serranía de Caparúch, de 4 a $5 \mathrm{~km}$ hacia el este $\left(14^{\circ} 35^{\prime} 04^{\prime \prime} \mathrm{S}\right.$; 6050'26" W; 190-195 m s.n.m .

El primer criterio de selección de los sitios es que fueran homogéneos fisonómicamente. En cada sitio se trazó una senda central de $500 \mathrm{~m}$, que fue demarcada con estacas cada 20 metros. De éstas, se seleccionaron 12 al azar y se sorteó la orientación respectiva (izquierda/derecha) donde se haría el levantamiento de datos (10 se realizaron en la época seca, y dos en la época de lluvias). A partir de cada estaca seleccionada, se trazó una línea de $22 \mathrm{~m}$, perpendicular a la senda principal, para el muestreo. Paralela a la línea, se abrió una senda con la finalidad de tener espacio para movilizarse y no alterar la estructura de la vegetación sobre la línea de muestreo. La toma de datos se hizo a partir de una distancia de $2 \mathrm{~m}$ de la senda principal, para dejar un callejón de acceso y amortiguación.

Se utilizaron tres métodos de muestreo. El primero es el punto de intercepción que sirvió para estratificar las diferentes formas de crecimiento en clases de altura (Mateucci y Colma, 1982). Este método consistió en utilizar una varilla delgada con escala graduada, la cual se colocó en forma vertical, registrando las plantas que se interceptaron a diferentes alturas. Se anotaron la forma de crecimiento (hierba, graminoide, subarbusto, arbusto, árbol, trepadora). Los puntos se establecieron a intervalos de un metro, sobre los $20 \mathrm{~m}$ de la línea de muestreo. Los datos fueron resumidos utilizando la siguiente fórmula:

\section{Cobertura Relativa $=(\mathrm{Ni} / \mathrm{Nt}) \times 100$}

donde:

$$
\begin{aligned}
\mathrm{Ni}= & \text { número de registros de cada } \\
& \text { forma de vida } \\
\mathrm{Nt}= & \text { número total de registros de to- } \\
& \text { das las plantas }
\end{aligned}
$$

De esta manera, se pudieron construir histogramas que representan la cobertura de cada una de las formas de vida, según las clases de altura. Para complementar la información sobre la estructura se realizaron diagramas de perfil en áreas representativas $(50 \times 10 \mathrm{~m})$ de cada sitio. En los perfiles vertical y horizontal se incluyen plantas con altura mayor a un metro, describiendo altura, forma y proyección de la copa.

El tercer método empleado fue el de líneas de intercepción, utilizado para obtener datos cuantita- 
tivos de cobertura y frecuencia de especies (Canfield, 1941; Smith, 1980); el muestreo se realizó sobre la misma línea donde se tomaron los datos de puntos de intercepción. Se midieron todas las intercepciones o proyecciones de las plantas (ramas, tallos, hojas, estructuras reproductivas) sobre la línea; en plantas macolladoras (graminoides) un individuo fue considerado como todo el conjunto de hijuelos que nacen de la base de la planta madre. En trepadoras, un individuo fue delimitado como el conjunto de todas las ramas que nacen en un mismo punto. La cobertura relativa se calculó por la siguiente fórmula:

Cobertura Relativa $=(\mathrm{Ie} / \mathrm{It}) \times 100$

donde:

$$
\begin{aligned}
\mathrm{Ie}= & \text { sumatoria de intercepción de } \\
& \text { cada especie } \\
\mathrm{It}= & \text { sumatoria de las intercepciones } \\
& \text { de todas las especies }
\end{aligned}
$$

La frecuencia de las especies mide su dispersión sobre un área. Para calcularla se registró la presencia o ausencia de cada especie en cada línea de muestreo. La frecuencia absoluta es el número total de estos registros de una especie en todas las líneas de muestreo (12); la frecuencia relativa es la relación de los registros absolutos de una especie y el número total de registros de todas las especies.

Se consideraron como hierbas a aquellas plantas anuales o perennes con tallos verduscos, de consistencia carnosa o jugosa, con alturas menores de $1.5 \mathrm{~m}$, y sin tejido leñoso. Las graminoides fueron aquellas plantas parecidas a las gramíneas (en este grupo se incluyeron a las Gramineae, Cyperaceae, Xyridaceae e Iridaceae). Los arbustos fueron aquellas plantas perennes con tejido leñoso, y que se ramifican desde la base. Subarbusto, es una terminología utilizada para aquellas plantas parecidas a arbustos, pero la diferencia

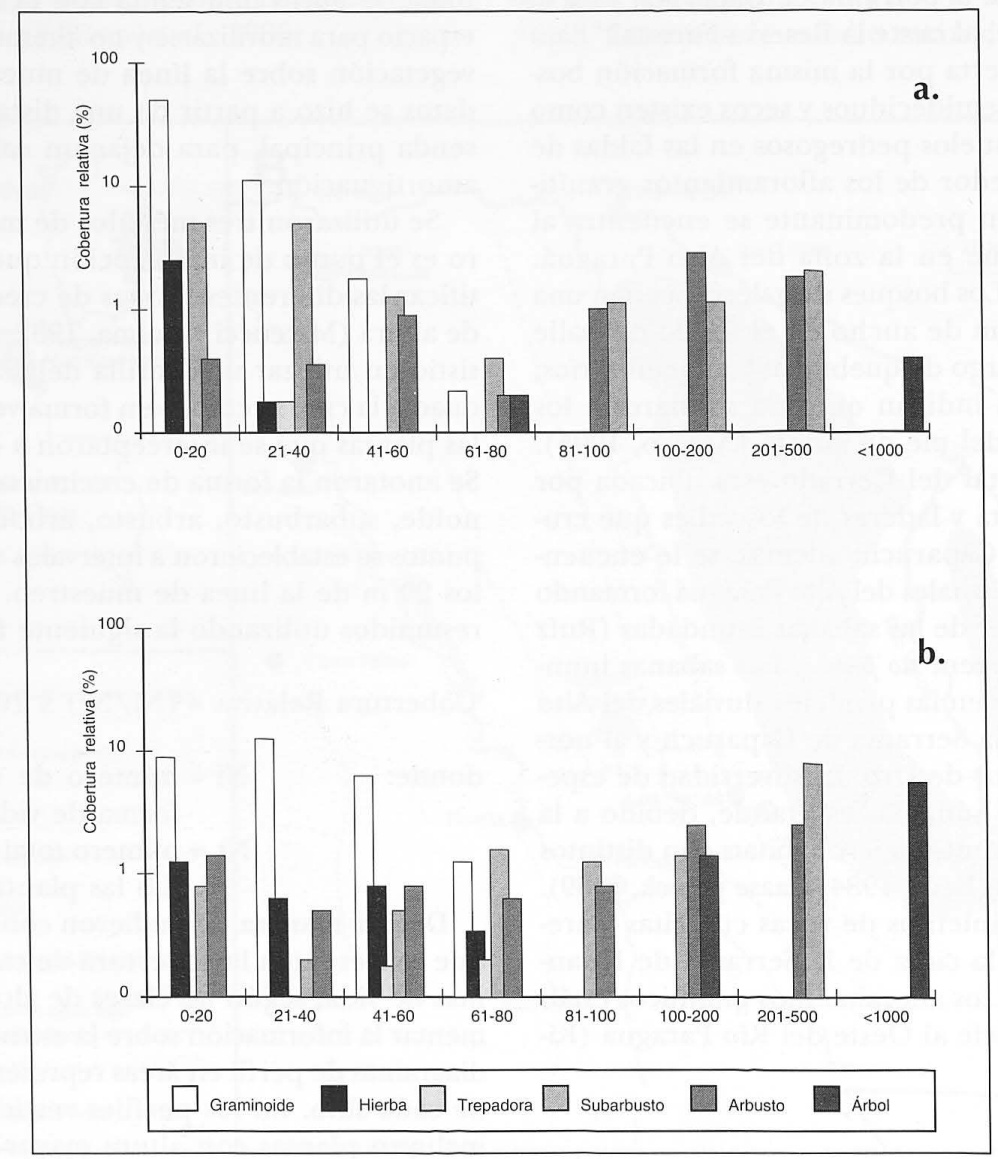

Figura 2. Representación gráfica de la relación entre clases de altura, cobertura y formas de crecimiento. Datos obtenidos por el método de puntos de intercepción. $\mathrm{a}=$ primer sitio, $\mathrm{b}=$ segundo sitio. 
esta en que solo la base de la planta tiene tejido leñoso. Los árboles son plantas perennes con tejido leñoso, pero con un eje principal en la base. Las trepadoras son aquellas plantas con o sin tejido leñoso que necesitan de otros individuos de diferente forma de crecimiento para sostenerse. En algunos casos, por las diferentes condiciones abióticas, una misma especie podía adquirir dos formas de crecimiento; para el análisis de los datos, se consideró aquella forma de crecimiento predominante.

Paralelamente a la toma de datos, se realizaron recolecciones de plantas, que en su mayoría fueron estériles. La identificación se realizó por comparación con muestras de herbario que fueron coleccionadas e identificadas durante el curso del proyecto Flora y Vegetación del PNK, de la cual este estudio es un componente. Un juego completo de ejemplares botánicos está depositado en el Herbario del Oriente Boliviano (USZ). Los duplicados han sido distribuidos a diferentes herbarios nacionales e internacionales, como el Herbario Nacional de Bolivia (LPB) y el Missouri Botanical Garden (MO). Todas las colectas de plantas realizadas en este trabajo están bajo la numeración del primer autor (BM).

Además, se realizaron perfiles de suelos en las diferentes parcelas de estudio, con la finalidad de comparar y relacionar con la vegetación. Los perfiles de suelo fueron clasificados tomando en cuenta características físico-químicas de los diferentes horizontes; la clasificación preliminar fue realizada utilizando la clave de suelos del sistema de la Organización Mundial de Alimentos (FAO, 1990).

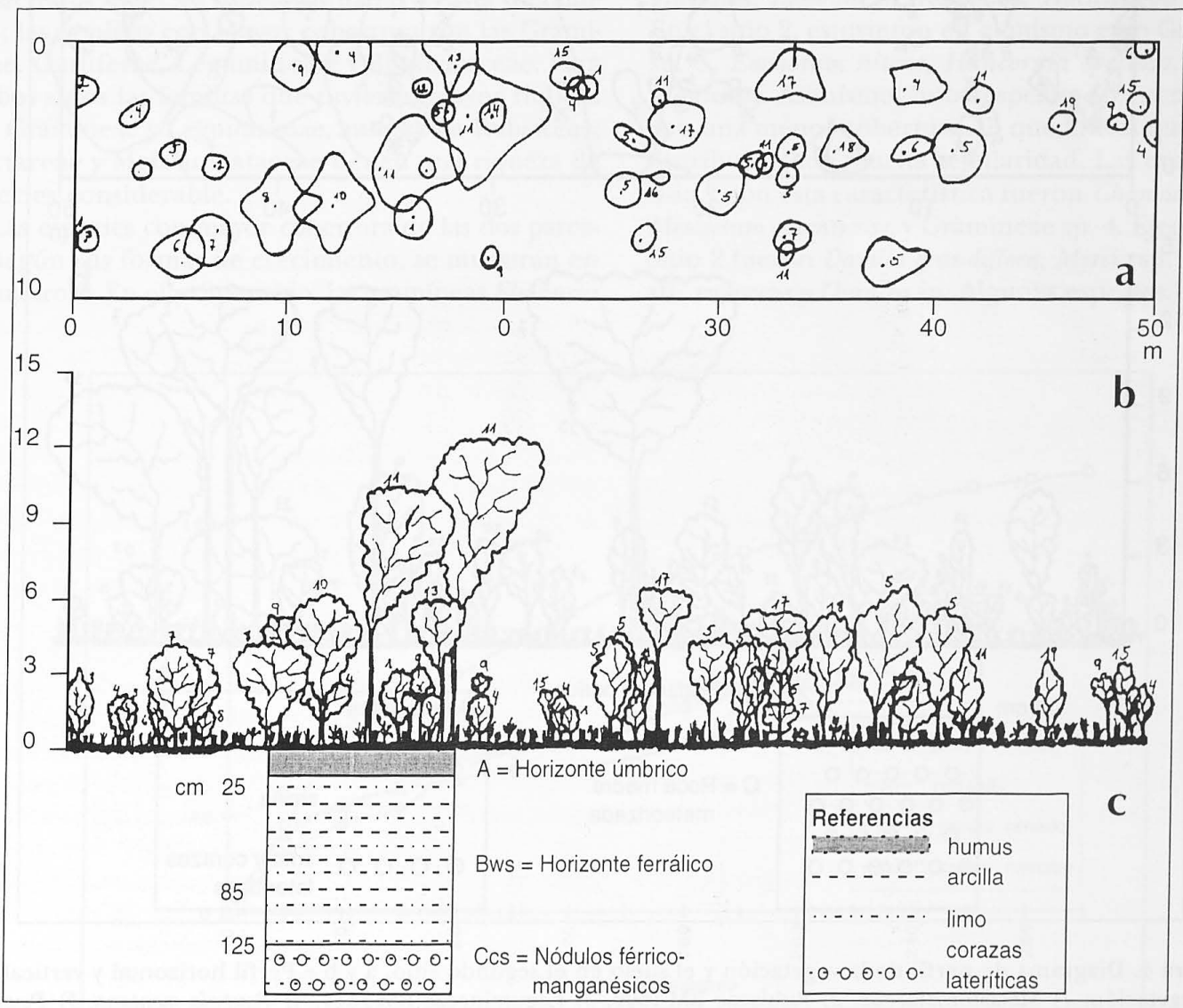

Figura 3. Diagramas de perfil de la vegetación y el suelo del primer sitio. a y b = Perfil horizontal y vertical de la vegetación: 1) Roupala montana, 2) Curatella americana, 3) BM-1572, 4) Pouteria ramiflora, 5) Byrsonima sp., 6) Lafoensia aff. vandelliana, 7) Caryocar brasiliense, 8) BM-1573, 9) Guttiferae BM-1574, 10) Bowdichia virgilioides, 11) Caraipa aff. densifolia, 12) Platypodium cf. elegans, 14) Dictychandra aurantiaca, 15) Psedobombax sp., 16) Connarus suberosus, 17) Tabebuia aff. aurea, 18) Mouriri sp., 19) Dictychandra aurantiaca; $\mathbf{c}=$ Perfil vertical del suelo. 


\section{Resultados}

Estructura de la vegetación. El Sitio 1 está ubicado en una zona con relieve uniforme y plano. La mayor cobertura $(90 \%)$ se encuentra en los estratos inferiores $(<40 \mathrm{~cm})$ donde las graminoides son las que predominan, pero también existe un importante componente de subarbustos y hierbas (figura $2 \mathrm{a}$ ). El estrato herbáceo es continuo y cubre el suelo en su totalidad. En los estratos superiores, los arbustos y árboles cuentan con una mayor cobertura, entre $100-500 \mathrm{~cm}$. Los árboles emergentes son pocos, pero llegan hasta $12 \mathrm{~m}$ de altura (figura $3 \mathrm{~b}$ ). En el perfil horizontal se observa claramente que en la mayoría de las copas de las plantas leñosas (subarbusto, arbusto, árbol) no llegan a juntarse, observándose predominio relativo del estrato herbáceo (figura 3a).

El Sitio 2 está situado a poca distancia del anterior, pero muestra una estructura distinta a pesar de formar parte del complejo del Cerrado. La figura $2 b$ muestra la distribución de la cobertura por formas de crecimiento según las distintas clases de altura. Más de la cobertura total $(59.2 \%)$ se encuentra por debajo de los $60 \mathrm{~cm}$, dominado por las graminoides, aunque también existe un importante componente de las demás formas de crecimiento. En el estrato

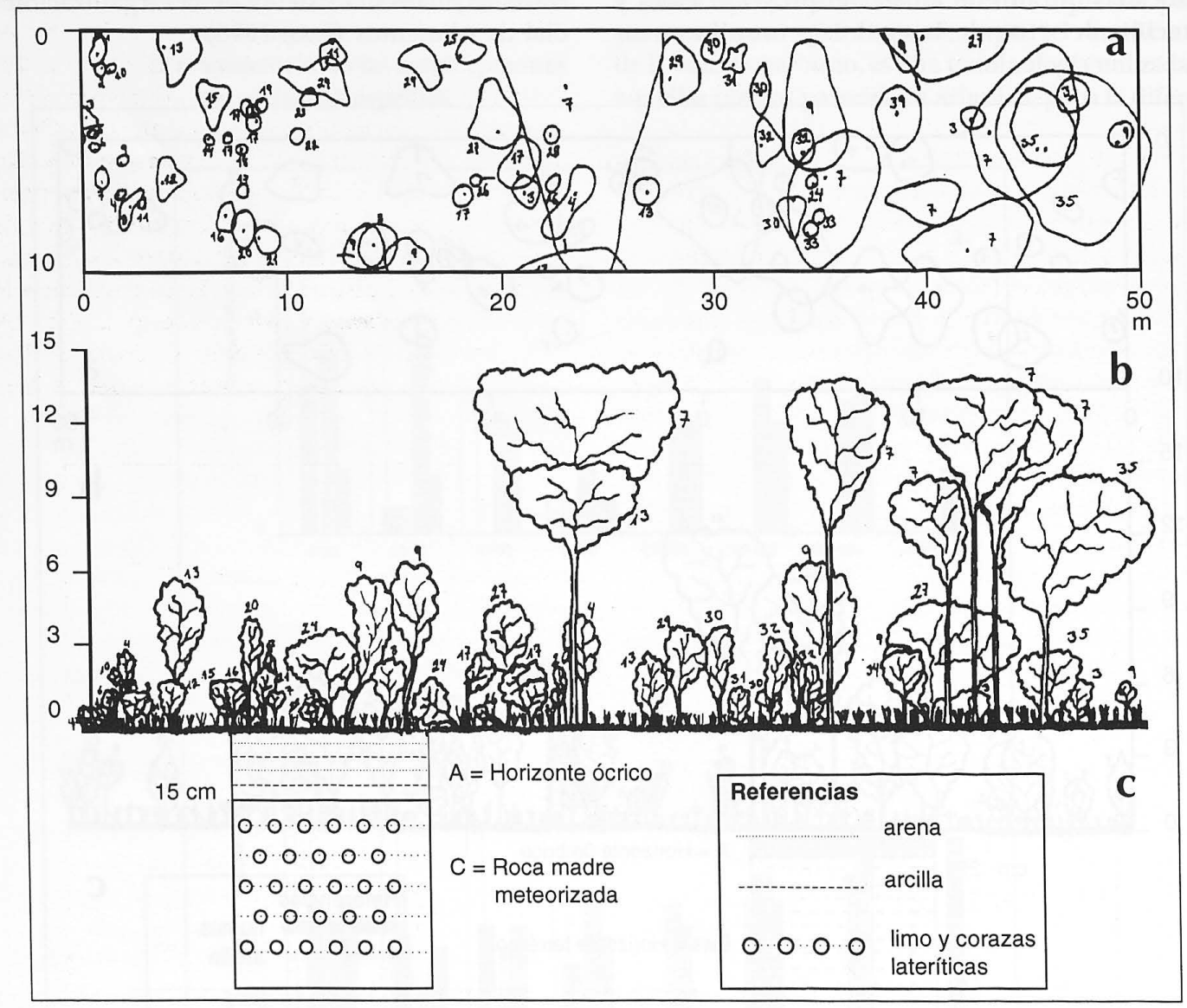

Figura 4. Diagrama de perfil de la vegetación y el suelo en el segundo sitio. a y $\mathrm{b}=$ Perfil horizontal y vertical de la vegetación: 1) Melastomataceae, 2) Rubiacae BM-1509, 3) Leguminosae BM-1511, 4) Roupala montana, 5) Pouteria ramiflora, 6) Myrtaceae, 7) Caraipa aff. densifolia, 8) Hancornia speciosa, 9) Mouriri cf. apiranga, 10) Erythroxylum, sp. 11) Rubiaceae BM-1509, 12) Melastomataceae, 13) Byrsonima sp., 14) Didimopanax distractiflorus, 15) Melastomataceae, 16) Leguminosae 1, 17) Myrtaceae, 18) Leguminosae 2, 19) Rubiaceae BM-1509, 20) Erythroxylum, 21) Myrtaceae 2, 22) Davilla grandiflora, 23) Kielmeyera, sp. 24) Erythroxylum, sp. 25) Leguminosae 4, 26) Hymatanthus sp., 27) Bombacaceae, 28) Neea aff. theifera, 29) Myrtaceae BM-1518, 30) Rubiaceae BM-1511, 31) Allagoptera leucocalyx, 32) Byrsonima aff. coccolobifolia, 33) Qualea sp., 34) BM-1519, 35) Lauraceae BM-1538; c = Perfil vertical del suelo. 
comp̀prendido entre 60 y $200 \mathrm{~cm}$ de altura, la mayor cobertura está compuesta por arbustos, con algunos individuos que pueden llegar a ser árboles. El estrato leñoso es discontinuo, si bien el perfil horizontal muestra la sobreposición de algunas copas de las plantas leñosas (figura 3a). La mayor cobertura de árboles se encuentra en alturas de 5 a $10 \mathrm{~m}$, aunque algunos llegan hasta $15 \mathrm{~m}$ (figura $4 \mathrm{~b}$ ).

Composición florística. El cuadro 1 presenta los datos de cobcrtura y diversidad para las familias más importantes registradas en los dos sitios. La primera parcela cuenta con 41 familias, 90 géneros y 112 especies (2 especies no fueron identificadas a nivel de familia); las familias con mayor cobertura son las Gramineae, Leguminosae, Rubiaceae y Myrtaceae. El segundo sitio cuenta con 42 familias, 89 géneros y 106 especies (7 especies indeterminadas a nivel de familia); las familias con mayor cobertura son las Gramineae, Guttiferae, Leguminosae y Bombacaceae. Para ambos sitios las familias que tuvieron mayor riqueza son Gramineae y Leguminosae, aunque las Rubiaceae, Myrtaceae y Melastomataceae tienen una riqueza de especies considerable.

Las especies con mayor cobertura de las dos parcelas según sus formas de crecimiento, se muestran en el cuadro 2. En el primer sitio, las gramíneas Elionurus muticus y Paspalum pectinatum (22.1\% y $10.7 \%$ ), tienen la mayor cobertura. En el segundo sitio, la gramínea Trachypogon plumosus (22.6\%) y el árbol Caraipa aff. densifolia (19.9\%), son las especies dominantes. Las especies más frecuentes de las dos parcelas según sus formas de crecimiento se muestran en el cuadro 3. Las más frecuentes en el primer sitio son Axonopus aff. marginatus, Ouratea spectabilis, Borreria sp., Paspalum pectinatum y Elionurus muticus. En cambio en el segundo sitio, Miconia albicans, Trachypogon plumosus, Paspalum pectinatum y Caraipa aff. densifolia, son las más frecuentes.

Hay varias especies con cobertura alta, pero con frecuencia baja, lo que indica que muchas de ellas crecen agrupadas en manchones o están representadas por árboles que tienen copas muy amplias. Entre estas, en el sitio 1 estuvieron Maytenus sp., Eriotheca aff. gracilipes, Tabebuia aff. roseo-alba, Trachypogon plumosus. En el sitio 2, estuvieron en el mismo caso Gramineae sp. 5, Emmotum nitens, Hancornia speciosa, Caryocar brasiliense. Asimismo, hubo especies frecuentes, pero con una menor cobertura, lo que indica que éstas se distribuyen con mucha regularidad. Las especies del sitio 1 con esta característica fueron Chamaecrista sp., Mesosetum cayennense, y Gramineae sp. 4. Ejemplos del Sitio 2 fueron Davilla grandiflora, Myrcia sp. 1, Smilax aff. rufescens y Ouratea sp. Algunas especies llaman la

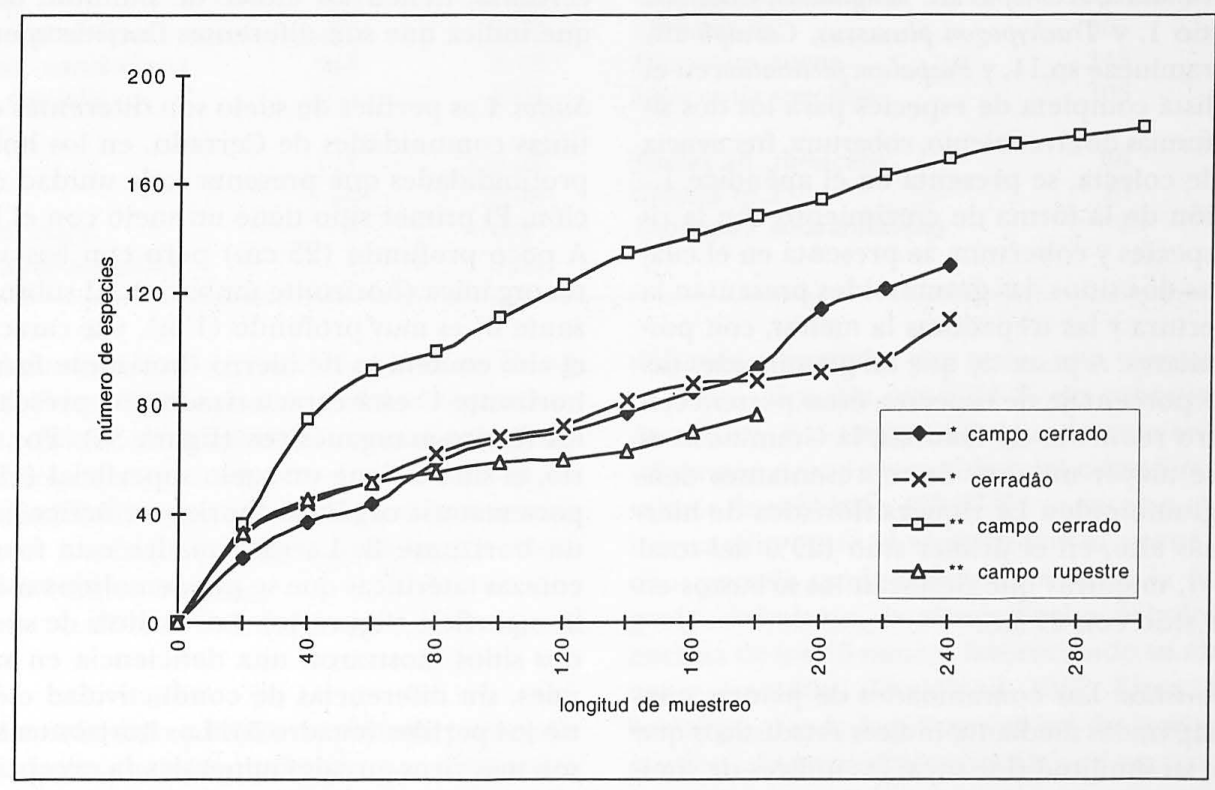

Figura 5. Diversidad florística en función de la longitud de la línea de muestreo para cuatro sitios en diferentes comunidades del Cerrado en el Parque Nacional "Noel Kempff Mercado". Los asteriscos indican: a) * las dos parcelas de estudio, y $b$ ) ** otras parcelas de estudio en el mismo Parque. Adaptado de Mostacedo et al., 1994. 
Cuadro 1. Familias más importantes en dos sitios diferentes con vegetación de cerrado en el Parque Nacional "Noel Kempff Mercado", Santa Cruz, Bolivia, ordenadas por su cobertura relativa (C.R.) y riqueza florística. GEN. = número de géneros, ESP. = número de especies.

\begin{tabular}{|c|c|c|c|c|c|c|}
\hline \multirow[t]{2}{*}{ Familia } & \multicolumn{3}{|c|}{ Sitio 1} & \multicolumn{3}{|c|}{ Sitio 2} \\
\hline & C.R. $(\%)$ & GEN. & ESP. & C.R. (\%) & GEN. & ESP. \\
\hline Gramineae & 50.80 & 15 & 19 & 51.00 & 18 & 19 \\
\hline Leguminosae & 5.71 & 11 & 15 & 4.83 & 11 & 11 \\
\hline Guttiferae & 1.69 & 2 & 3 & 20.38 & 2 & 3 \\
\hline Rubiaceae & 5.04 & 4 & 4 & 0.39 & 4 & 6 \\
\hline Myrtaceae & 4.35 & 3 & 7 & 1.67 & 2 & 6 \\
\hline Melastomataceae & 0.07 & 2 & 2 & 3.18 & 3 & 5 \\
\hline Dilleniaceae & 3.85 & 2 & 2 & 0.85 & 1 & 1 \\
\hline Celastraceae & 3.26 & 1 & 1 & 0.42 & 1 & 1 \\
\hline Malpighiaceae & 3.04 & 2 & 3 & 1.15 & 2 & 2 \\
\hline Bombacaceae & 2.95 & 2 & 4 & 2.21 & 2 & 2 \\
\hline Lythraceae & 2.23 & 2 & 2 & 0.11 & 3 & 3 \\
\hline Óchnaceae & 1.96 & 1 & 1 & 0.44 & 1 & 2 \\
\hline Proteaceae & 1.90 & 1 & 1 & 2.12 & 1 & 1 \\
\hline Caryocaraceae & 1.34 & 1 & 1 & 1.33 & 1 & 1 \\
\hline Otras & 11.81 & 41 & 47 & 9.96 & 37 & 43 \\
\hline Totales & 100.00 & 90 & 112 & 100.00 & 89 & 106 \\
\hline
\end{tabular}

atención porque tuvieron tanto coberturas como frecuencias altas, tal fue el caso de Elionurus muticus, Paspalum pectinatum, Axonopus aff. marginatus, y Borreria sp. en el Sitio 1, y Trachypogon plumosus, Caraipa aff. densifolia, Gramineae sp.11, y Paspalum pectinatum en el Sitio 2. La lista completa de especies para los dos sitios con sus formas de crecimiento, cobertura, frecuencia y número de colecta, se presenta en el apéndice 1 .

La relación de la forma de crecimiento con la riqueza de especies y cobertura, se presenta en el cuadro 4. En los dos sitios, las graminoides presentan la mayor cobertura y las trepadoras la menor, con porcentajes similares. A pesar de que las graminoides tienen un alto porcentaje de especies, éstas pertenecen a un número reducido de familias; la Gramineae es la que tiene mayor número de representantes dentro de las graminoides. La riqueza florística de hierbas es el más alto, en el primer sitio ( $29 \%$ del total de especies), mientras que destacan los arbustos en el segundo sitio con $27 \%$.

Similitud florística. Las comunidades de plantas pueden ser comparadas mediante índices estadísticos que cuantifican su similitud florística; los índices de similitud expresan la semejanza de dos o más registros de inventarios de vegetación. Para este trabajo se utilizó el índice de Sørensen (Matteucci y Colma, 1982; Magurran, 1989). Del total de 189 especies registra- das en los dos sitios, sólo 33 especies están presentes en las dos áreas, las cuales a pesar de ser relativamente cercanas, tienen un índice de similitud de $39 \%$, lo que indica que son diferentes florísticamente.

Suelos. Los perfiles de suelo son diferentes en las distintas comunidades de Cerrado, en los horizontes y profundidades que presenta cada unidad de vegetación. El primer sitio tiene un suelo con el horizonte A poco profundo $(25 \mathrm{~cm})$ pero con bastante materia orgánica (horizonte úmbrico). El subsuelo (horizonte B) es muy profundo ( $1 \mathrm{~m})$, y se caracteriza por el alto contenido de hierro (horizonte ferrálico). El horizonte $\mathrm{C}$ está caracterizado por presentar nódulos férrico-manganésicos (figura 3c). Por el contrario, el sitio 2 tiene un suelo superficial $(15 \mathrm{~cm})$ con poca materia orgánica (horizonte ócrico); carece de un horizonte B. La roca madre está formada por corazas lateríticas que se pueden observar incluso en la superficie (figura 4c). Los análisis de suelos en los dos sitios mostraron una deficiencia en sales minerales, sin diferencias de conductividad eléctrica entre los perfiles (cuadro 5). Los horizontes superiores son más ricos en sales minerales (a excepción del Ferrasol plíntico), debido a la humificación de materia orgánica en estos horizontes. Todos estos suelos son ácidos, lo que puede estar relacionado a la lixiviación de nutrientes (Furley y Ratter, 1988). La dis- 
Cuadro 2. Cobertura absoluta (CA) y relativa (CR) de las especies más dominantes clasificadas por su forma de crecimiento, en dos sitios del Cerrado en el Parque Nacional "Noel Kempff Mercado", Santa Cruz, Bolivia.

\begin{tabular}{|c|c|c|c|c|c|}
\hline \multicolumn{3}{|c|}{ Sitio 1} & \multicolumn{3}{|c|}{ Sitio 2} \\
\hline Especie & CA & CR (\%) & Especie & $\mathrm{CA}$ & CR (\%) \\
\hline \multicolumn{6}{|l|}{ Graminoides } \\
\hline Elionurus muticus & 2718 & 22.12 & Trachypogon plumosus & 3737 & 22.56 \\
\hline Paspalum pectinatum & 1312 & 10.68 & Graminae sp. 11 & 1953 & 11.79 \\
\hline Axonopus aff. marginatus & 849 & 6.91 & Graminae sp. 5 & 732 & 4.42 \\
\hline Digitaria dioica & 357 & 2.91 & Paspalum pectinatum & 497 & 3.00 \\
\hline \multicolumn{6}{|l|}{ Hierbas } \\
\hline Borreria sp. & 599 & 4.88 & Reincourtia aff. oblongifolia & 73 & 0.44 \\
\hline Hyptis sp. 2 & 54 & 0.44 & Manihot caurulescens & 21 & 0.13 \\
\hline Chamaecrista? sp. & 52 & 0.42 & Crotalaria? sp. & 15 & 0.09 \\
\hline Helicteres sp. 1 & 43 & 0.35 & Compositae sp. 4 & 14 & 0.08 \\
\hline \multicolumn{6}{|l|}{ Subarbustos } \\
\hline Psidium salutare & 330 & 2.69 & Myrcia guianensis & 80 & 0.48 \\
\hline Ouratea spectabilis & 240 & 1.96 & Matayba guianensis & 80 & 0.48 \\
\hline Bahuinia rufa & 235 & 1.91 & Aspidosperma? sp. & 50 & 0.30 \\
\hline Tetrapteris ambigua & 101 & 0.82 & Ouratea sp. & 44 & 0.27 \\
\hline \multicolumn{6}{|l|}{ Arbustos } \\
\hline Davilla grandiflora & 465 & 3.78 & Miconia albicans & 418 & 2.52 \\
\hline Roupala montana & 233 & 1.90 & Roupala montana & 352 & 2.12 \\
\hline Casearia sylvestris & 102 & 0.83 & Bowdichia virgilioides & 220 & 1.33 \\
\hline $\begin{array}{l}\text { Hyeronima sp. } 1 \\
\text { Árboles }\end{array}$ & 61 & 0.50 & Byrsonima crassifolia & 180 & 1.09 \\
\hline Maytenus sp. & 400 & 3.26 & Caraipa aff. densifolia & 3293 & 19.88 \\
\hline Eriotheca cf. gracilipes & 310 & 2.52 & Ormosia sp. & 385 & 2.32 \\
\hline Lafoensia aff. vandelliana & 268 & 2.18 & Emmotum nitens & 345 & 2.08 \\
\hline Byrsonima fagifolia & 253 & 2.06 & Hancornia speciosa & 305 & 1.84 \\
\hline \multicolumn{6}{|l|}{ Trepadoras } \\
\hline Clitoria sp. 1 & 11 & 0.09 & Smilax aff. rufescens & 68 & 0.41 \\
\hline Smilax sp. & 10 & 0.08 & Clitoria sp.2 & 20 & 0.12 \\
\hline Serjania reticulata & 9 & 0.07 & Dioscorea amaranthoides & 2 & 0.01 \\
\hline Dioscorea amaranthoides & 7 & 0.06 & & & \\
\hline
\end{tabular}

ponibilidad de elementos según el diagrama de Truog (Roquero \& Porta, 1986), indica que son suelos metalotóxicos, donde el aluminio, cobre, manganeso, boro y zinc son bastante solubles a estos valores de $\mathrm{pH}$. Además, el fósforo, potasio, calcio y magnesio son poco solubles y menos accesibles para las plantas a estos mismos valores de $\mathrm{pH}$.

\section{Discusión}

Estructura. La cobertura y altura de plantas, principalmente de árboles, ha sido utilizada por diferentes autores para separar las distintas categorías de Cerrado (sensu lato; cuadro 6). Utilizando los criterios de Goodland (1969) y Eiten (1990), el primer sitio se puede clasificar como un ejemplo de la comunidad vegetal del campo cerrado, por tener una vegetación abierta y baja (árboles con 3-14 \% de cobertura y 1-5 m de altura). En cambio, el segundo sitio se puede clasificar como una comunidad del cerradâo; si bien los valores de cobertura (22-27 \%) se encajan en un punto intermedio entre el cerrado (sensu stricto) y el cerradâo, pero la tendencia apunta hacia la última categoría. Asimismo, la altura de los árboles está por encima de los 15 metros favoreciendo su clasificación como cerradâo, (Goodland, 1969; Eiten, 1990). Sin embargo, por la heterogeneidad de la vegetación se pueden observar algunas zonas con características de cerrado (sensu stricto).

Aunque la cobertura total de las graminoides en los dos sitios es similar, están distribuidas en porcentajes diferentes de clases de altura. Así, en el sitio 1 
Cuadro 3. Lista de las especies más frecuentes clasificadas por su forma de crecimiento, en dos sitios del Cerrado en el Parque Nacional "Noel Kempff Mercado", Santa Cruz, Bolivia.

$\mathrm{FA}=$ frecuencia absoluta, $\mathrm{FR}=$ frecuencia relativa.

\begin{tabular}{|c|c|c|c|c|c|}
\hline \multicolumn{3}{|l|}{ Sitio 1} & \multicolumn{3}{|l|}{ Sitio 2} \\
\hline Especie & FA & FR (\%) & Especie & FA & FR $(\%)$ \\
\hline \multicolumn{6}{|l|}{ Graminoides } \\
\hline Axonopus aff. marginatus & 12 & 3.45 & Trachypogon plumosus & 11 & 4.03 \\
\hline Paspalum pectinatum & 11 & 3.16 & Paspalum pectinatum & 10 & 3.66 \\
\hline Elionurus muticus & 11 & 3.16 & Gramineae sp. 6 & 9 & 3.30 \\
\hline Digitaria dioica & 10 & 2.87 & Digitaria dioica & 9 & 3.30 \\
\hline \multicolumn{6}{|l|}{ Hierbas } \\
\hline Borreria sp. & 11 & 3.16 & Reincourtia aff. oblongifolia & 5 & 1.83 \\
\hline Chamaecrista sp. & 8 & 2.30 & Manihot caurulescens & 5 & 1.83 \\
\hline Chamaecrista desvauxii & 5 & 1.44 & Adiantum sp. 2 & 2 & 0.73 \\
\hline Helicteres sp. & 5 & 1.44 & Compositae sp.3 & 2 & 0.73 \\
\hline \multicolumn{6}{|l|}{ Subarbustos } \\
\hline $\begin{array}{l}\text { Ouratea spectabilis } \\
\text { Psidium salutare }\end{array}$ & $\begin{array}{l}12 \\
10\end{array}$ & 3.44 & Myrcia sp.1 & 8 & 2.93 \\
\hline $\begin{array}{l}\text { Psidium salutare } \\
\text { Bahuinia rufa }\end{array}$ & 10 & 2.87 & Ouratea sp. & 6 & 2.20 \\
\hline Bahuinia rufa & 6 & 1.72 & Erythrxylum suberosum & 4 & 1.47 \\
\hline Tetrapteris ambigua & 5 & 1.44 & Matayba guianensis & 3 & 1.10 \\
\hline \multicolumn{6}{|l|}{ Arbustos } \\
\hline Davilla grandiflora & 9 & 2.59 & Miconia albicans & 12 & 4.40 \\
\hline Roupala montana & 5 & 1.44 & Roupala montana & 8 & 2.93 \\
\hline Casearia sylvestris & 4 & 1.15 & Davilla grandiflora & 8 & 2.93 \\
\hline $\begin{array}{l}\text { Erythroxylum aff. angifugum } \\
\text { Árboles }\end{array}$ & 3 & 0.86 & Casearia javitensis & 4 & 1.47 \\
\hline Caraipa aff. densifolia & 7 & 2.01 & Caraipa aff. densifolia & 10 & 3.66 \\
\hline Byrsonima aff. orbigniana & 7 & 2.01 & Ormosia sp. & 7 & 2.56 \\
\hline Pouteria aff. ramiflora & 5 & 1.44 & Pouteria aff. ramiflora & 6 & 2.20 \\
\hline Lafoensia vandelliana & 4 & 1.15 & Caryocar brasiliense & 3 & 1.10 \\
\hline \multicolumn{6}{|l|}{ Trepadoras } \\
\hline Clitoria sp. 2 & 4 & 1.15 & Smilax aff. rufescens & 7 & 2.56 \\
\hline Clitoria sp.1 & 2 & 0.57 & Clitoria sp. 2 & 1 & 0.37 \\
\hline Dioscorea amaranthoides & 1 & 0.29 & Dioscorea amaranthoides & 1 & 0.37 \\
\hline Serjania reticulata & 1 & 0.29 & & & \\
\hline
\end{tabular}

(campo cerrado), la mayor cobertura está entre los 20 y 40 cm; en cambio, en el sitio 2 (cerradâo) está entre 0 y $20 \mathrm{~cm}$ de altura. La estructura representada en los histogramas (figura 2) es diferente en los dos sitios, y también es distinta a los otros sitios estudiados en el PNK, el campo rupestre y el campo cerrado de Las Gamas, donde la cobertura de árboles es menor en comparación a los sitios de este estudio (Mostacedo et al., 1994).

Estas variaciones pueden ser el resultado de factores que influyen y actúan sobre las distintas comunidades del Cerrado. Por ejemplo, la topografía del terreno del sitio 1 (campo cerrado) es plana y baja, mientras que el sitio 2 (cerradâo) tiene un relieve más complejo, con pendientes entre 3 y $5^{\circ}$. El sitio 1 , tie- ne un suelo más profundo y presenta un horizonte A con bastante materia orgánica y con plintita a una profundidad de $125 \mathrm{~cm}$ a partir de la superficie (ferrasol plíntico-húmico). Por el contrario, el sitio 2 tiene un suelo superficial con poca materia orgánica, que está limitado por la profundidad de la roca madre (leptosol dístrico; figura 4c). Otro factor que parece influir en la diferencia entre estas dos áreas, principalmente en la estructura de la vegetación, es la quema anual (Eiten, 1990); el sitio 1 presenta indicios de quema antropogénica en la época seca, ocasionada probablemente por estar próximo a una carretera. En cambio, el segundo sitio parece no ser quemado durante péríodos más largos. La diferencia en la cantidad de carbón en la corteza y rizomas de las plan- 
Cuadro 4. Datos de riqueza florística y cobertura relativa para cada forma de crecimiento de las especies en dos sitios de estudio. FAM. = número de familias, ESP.= número de especies, C.R. $=$ porcentaje de cobertura relativa obtenido por el método de líneas de intercepción.

\begin{tabular}{|c|c|c|c|c|c|c|}
\hline \multirow[t]{2}{*}{ Familia } & \multicolumn{3}{|c|}{ Sitio 1} & \multicolumn{3}{|c|}{ Sitio 2} \\
\hline & FAM & ESP. & C.R. (\%) & FAM & ESP. & C.R. (\%) \\
\hline Graminoide & 4 & 24 & 51.15 & 2 & 22 & 50.86 \\
\hline Hierba & 17 & 33 & 7.93 & 15 & 28 & 13.24 \\
\hline Trepadora & 4 & 5 & 0.40 & 3 & 3 & 0.50 \\
\hline Subarbusto & 8 & 15 & 10.90 & 8 & 13 & 2.68 \\
\hline Arbusto & 12 & 16 & 9.11 & 20 & 29 & 11.37 \\
\hline Árbol & 14 & 22 & 19.66 & 12 & 14 & 32.45 \\
\hline Totales & 59 & 112 & 100.00 & 60 & 106 & 100.00 \\
\hline
\end{tabular}

Cuadro 5. Algunas características físico-químicas de los suelos del área de estudio. C.E.= conductividad eléctrica, $\mathrm{mS}=$ mili-Siemens, mmhos=milimohos, meq./l=miliequivalentes por litro, $A=$ arena, $L=l i m o, Y=a r c i l l a$, Aum=horizonte $\mathrm{A}$ úmbrico, Aoc=horizonte $\mathrm{A}$ ócrico, $\mathrm{Bws}=$ horizonte $\mathrm{B}$ ferrálico, $\mathrm{C}=$ horizonte $\mathrm{C}$ con roca madre meteorizada, $\mathrm{Ccs}=$ horizonte $\mathrm{C}$ con nódulos férrico-manganésicas.

\begin{tabular}{|c|c|c|c|c|c|c|}
\hline \multirow[t]{2}{*}{ Propiedades } & \multicolumn{3}{|c|}{$\begin{array}{c}\text { Ferrasol plíntico } \\
\text { Sitio } 1\end{array}$} & \multicolumn{3}{|c|}{$\begin{array}{c}\text { Leptosol dístrico } \\
\text { Sitio } 2\end{array}$} \\
\hline & Aum & Bws1 & Bws2 & Ccs & Aoc & C \\
\hline \multicolumn{7}{|l|}{ C.E. } \\
\hline $\mathrm{mS}(1: 1)=\mathrm{mmhos}$ & 0.05 & 0.07 & 0.07 & 0.04 & 0.05 & 0.03 \\
\hline meq./l & 0.5 & 0.7 & 0.7 & 0.4 & 0.5 & 0.3 \\
\hline $\mathrm{pH}$ (agua) $1: 1$ & 5.0 & 4.9 & 4.9 & 5.0 & 4.9 & 4.8 \\
\hline Textura & $\mathrm{AL}$ & LY & Y & Y & $\mathrm{AL}$ & LY \\
\hline
\end{tabular}

Cuadro 6. Clasificación de comunidades vegetales de la formación fitogeográfica del Cerrado, utilizando la cobertura y altura de árboles como criterios principales. A=Goodland (1969), B=Eiten (1990).

\begin{tabular}{|c|c|c|c|c|c|c|}
\hline \multirow{2}{*}{$\begin{array}{l}\text { Parámetros de } \\
\text { clasificación }\end{array}$} & \multicolumn{2}{|c|}{ Campo cerrado } & \multicolumn{2}{|c|}{ Cerrado } & \multicolumn{2}{|c|}{ Cerradâo } \\
\hline & A & B & A & B & A & B \\
\hline Cobertura (Prom.) & 3 & 3 & 19 & 20 & 46 & 30 \\
\hline Árboles (\%) (rango) & $0-15$ & & $1-55$ & & $15-85$ & \\
\hline Altura general (Prom.) & 4 & & 6 & & 9 & \\
\hline De árboles (rango) & $3-6$ & & $4-8$ & & $6-18$ & $7-15$ \\
\hline
\end{tabular}


tas permitió estimar que el sitio 1 podría tener una alteración por fuego más frecuente.

Diversidad y composición floristica. El número de especies registrado en relación a la longitud del muestreo (análisis análogo a una curva especie/área), determina la representatividad florística del muestreo (figura 5). En la época de lluvias hubo un incremento bajo en el registro de especies, principalmente de pastos y hierbas que no aparecieron en el primer muestreo debido a su estado fenológico. Este fenómeno fue más notable en el primer sitio donde hubo un incremento de $17 \%$ del total de especies, en comparación con el segundo donde el incremento fue sólo del $12 \%$. Una comparación con otros sitios con vegetación similar en el PNK muestra que el número de especies en campo cerrado (Las Gamas) tiende a estabilizarse a los $300 \mathrm{~m}$. Asimismo, en el campo rupestre (Las Gamas), los $180 \mathrm{~m}$ de línea muestreada fueron suficientes para lograr un muestreo completo (Mostacedo et al., 1994; figura 5). También se puede señalar que en las tres primeras líneas, se muestrearon una proporción considerable de especies (campo cerrado: $47 \%$; cerradâo: $34 \%$ ) respecto al total de especies; en cambio, en las parcelas de Las Gamas, en las tres primeras líneas se muestrearon más de la mitad del total de especies (campo cerrado: $50 \%$; campo rupestre: $67 \%$ ). Por otra parte, el campo cerrado de las Gamas es obviamente más rico en especies; posiblemente esta zona tiene una vegetación representativa de la formación del Cerrado, por estar en la cima de la serranía, mientras que los cerrados de las llanuras aluviales del Alto Paraguá podrían presentar las especies más comunes y con amplia distribución (Killeen, com. pers.) Los datos y las observaciones visuales indican que las líneas de muestreo no han interceptado a muchas especies que han sido recolectadas en la misma zona; estas observaciones nos permiten sugerir que la longitud de la línea muestreada no fue suficiente.

Aunque la correlación existente entre la cobertura y el número de especies por familia es alta y positiva (sitio 1, r=0.82; sitio 2, r= $=0,75$ ), en algunos casos esta relación no se cumple. En el primer sitio, las Gramineae tienen una cobertura relativa mayor al $50 \%$ para 19 especies; en cambio, las Leguminosae cuentan con una cobertura relativa entre 5 y $6 \%$, pero están representadas por 15 especies. La razón de este patrón de abundancia y diversidad parece ser que las Gramineae presentan especies con macollos grandes que predominan sobre otras especies, mientras que las Leguminosae son principalmente especies herbáceas de porte bajo. La alta riqueza de las leguminosas probablemente se debe a que tienen representantes en todas las formas de crecimiento. Otro fenómeno similar al anterior se presenta en el sitio 2, donde las Guttiferae, con solo tres especies, tuvieron el $20.3 \%$ de cobertura relativa; en este caso, esta familia cuenta con una especie arbórea (Caraipa aff. densifolia) que es la planta más abundante (19.9\%) dentro de esta forma de crecimiento.

Las familias más diversas en los dos sitios son Leguminosae y Gramineae. Esta observación coincide con otros estudios que destacan que las Myrtaceae y Rubiaceae cuentan también con una diversidad alta (Goodland, 1970; Heringer et al., 1977; Mantovani et al., 1985). El número total de especies (189) encontrados en los dos sitios representan sólo una parte de lo que conforma el Cerrado. Una lista preliminar de especies del Cerrado para el PNK realizada por Killeen (no publ.) menciona 501 especies. Por su parte Mantovani et al. (1985) cita a 96 géneros y 129 especies en el Cerrado; mientras que Goodland (1970) menciona 319 géneros y 515 especies. Al parecer, estas cifras son una subestimación de la diversidad del Cerrado. Una comparación de las diferentes listas florísticas (Mantovani, 1985; Heringer et al., 1977; Killeen y Nee, 1991) indica que los sitios de estudio tienen menos del $35 \%$ de especies en común. Esta poca semejanza puede estar relacionada con las diferencias de tipos de suelos de las comunidades vegetales. Para verificar la similitud entre dos comunidades se deberían tomar en cuenta también datos de cobertura y frecuencia, parámetros que no se tomaron en cuenta en el índice de Sørensen. Asimismo, el índice obtenido es bajo, porque posiblemente el muestreo es todavía insuficiente y existen especies que aún no han sido registradas.

En conclusión podemos decir que los dos sitios estudiados tienen especies que son propias del Cerrado (sensu lato) y se diferencian por la cobertura y altura de árboles. El sitio 1 estaría clasificado como el campo cerrado y el sitio 2 como cerradâo, aunque algunas partes corresponden al cerrado (sensu stricto).

\section{Agradecimientos}

El estudio se realizó gracias al apoyo financiero de la National Science Foundation (NSF), United States Agency for International Development (USAID) y el Fondo Nacional del Medio Ambiente (FONAMA), Bolivia. El artículo fue revisado por el Dr. Miguel Martínez Ramos y el Dr. Jorge Meave del Castillo, ambos profesores e investigadores de la Universidad Nacional Autónoma de México. La identificación de muchas plantas fue realizada por Luzmila Arroyo y Enrique Gutierrez, ambos investigadores del Museo de Historia Natural Noel Kemmpff Mercado. 


\section{Literatura citada}

Arroyo P. L. 1995. Estructura y composición de una isla de bosque y un bosque de galería en el Parque Nacional Noel Kempff Mercado. Tesis de licenciatura. Universidad Autónoma "Gabriel René Moreno". Santa Cruz, Bolivia.

Beck S. G. 1984. Comunidades vegetales de las sabanas inundadas de NE de Bolivia. Phytocoenologia 12:321-350

Beck S. G., T. J. Killeen y E. García E. 1993. Vegetación de Bolivia. En: Killeen, T. J., E. García E. y S. G. Beck eds. Guia de árboles de Bolivia. Herbario Nacional de Bolivia y Missouri Botanical Garden. 6-24.

Canfield R. 1941. Aplication of the Line-intercept method in sampling range vegetation. Forestry 39:388-396

CORDECRUZ-KFW. 1992. Mapa de Isoyetas e Isotermas, Santa Cruz-Bolivia. Escala 1: 4.000.000. Proyecto de Protección de Recursos Naturales.

Coutinho M. L. 1978a. O conceito do cerrado. Revista Brasileira do Botánica 1:17-23.

Coutinho M. L. 1978b. Aspectos ecológicos do fogo no cerrado. I.- A temperatura do solo durante as quemadas. Revista Brasileira do Botánica 1:93-96.

Di Stéfano G.J.F., L. Brenez y V. Mora. 1995. Composición florística y estructura de un bosque primario del piso premontano pluvial en San Ramón, Costa Rica. Revista de Biología Tropical 43:67-73.

Eiten G. 1972. The Cerrado Vegetation of Brazil. Botanical Review 38:201-341.

Eiten G. 1990. Vegetation. En: Novaes, P.M. (ed.). Cerrado: Caracterizacao, ocupacao e perspectivas. Editora Universidade de Brasilia. Brasilia, Brasil, 9-65.

FAO. 1990. Mapa mundial de suelos. Leyenda revisada. Roma, Italia. 142 pp.

Furley P. A. y J. A. Ratter. 1988. Soil resources and plant communities of the central Brazilian cerrado and their development. Journal of Biogeography 15:97-108.

Goodland R. J. A. 1969. An Ecological Study of the Cerrado Vegetation of South Central Brasil. Tesis de Ph. D., McGill University, Montreal, Canadá. 224 pp.

Goodland R. J. A. 1970. Plants of the Cerrado Vegetation of Brasil. Phytologia 20:57-78

Haase R. y S. Beck. 1989. Structure and composition of savanna vegetation in northern Bolivia: a preliminary report. Brittonia 41:80-100.

Heringer E. P., M. G. Barroso, A. J. Rizzo y T. C. Rizzini. 1977. A Flora do cerrado. En: Ferri, M. G. (ed.) IV Simposio sobre o cerrado, bases para utilizacâo agropecuaria. Editora da Universidade de Sao Paulo, Livraria, Itatiaia Editora Lta., Sao Paulo.

Killeen T. J., B. T. Louman y T. Grimnwood. 1990. La Ecología paisajística de la región de Concepción y Lomerío en la provincia Nuflo de Chavez, Santa Cruz. Ecología en Bolivia 16:1-45.

Killeen T. J. y M. Nee. 1991. Catálogo de las plantas saba- neras de Concepción, Depto. de Santa Cruz, Bolivia. Ecología en Bolivia 17:53-7.

Killeen T. J., E. García E. y S. G. Beck. 1993. Guía de árboles de Bolivia. Herbario Nacional de Bolivia y Missouri Botanical Garden. La Paz, Bolivia. 958 pp.

Mantovani W., Leitao F. H. y F. Martins. 1985. Chave baseada en caracteres vegetativos para identificacao de especies lenhosas do cerrado da reserva biológica de Moji Gua$\mathrm{cu}$, Estado de Sao Paulo. Hoehnea. 12:35-56.

Magurran A. E. 1989. Diversidad ecológica y su medición. Ediciones Vedra. España. 200 pp.

Matteuci S. y A. Colma 1982. Metodología para el Estudio de la Vegetación. Secretaria General de la Organización de los Estados Americanos. Washington, D.C.-U.S.A. Serie de Biología No 22. 162 pp.

Moraes M. y S. G. Beck. 1992. Diversidad florística de Bolivia. Cap. 3. En: Marconi, M. (ed.). Conservación de la diversidad biológica en Bolivia. Centro de datos para la conservación (CDC-BOLIVIA) y United States aid mision to Bolivia (USAID-BOLIVIA). 75-111 pp.

Mostacedo C. B., R. Guillen. y T. J. Killeen. 1994. Diversidad Florística de las Sabanas del Cerrado del Parque Nacional “ Noel Kempff Mercado”, Santa Cruz, Bolivia. VI Congreso Latinoamericano de Botánica, Mar del PlataArgentina.

Navarro G. 1992. Proyecto de protección de los recursos naturales en el departamento de Santa Cruz. Estudio de parques nacionales y otras áreas protegidas (informe). CORDECRUZ-KFW-CONSORCIO IP/SCG/KWC. Santa Cruz de la Sierra, Bolivia. 114 pp.

Navarro G. 1994. Avance sobre un modelo integrado de sectorización biogeográfica de Bolivia. Rev. Soc. de Est. Bot. 1 (1):40-48.

Novaes P. M. (ed.) 1990. Cerrado: Caracterizacao, ocupacao e perspectivas. Editora Universidade de Brasilia. Brasilia, Brasil. 657 pp.

Roche M. A. y N. Rocha. 1985. Precipitaciones anuales. PHICAB: SENAMHI-ORSTOM. La Paz: 1 mapa.

Roquero C. y J. Porta. 1986. Agenda de campo para estudio del suelo. 4ta. ed. Universidad Politécnica de Madrid. Madrid, España. 192 pag.

Ruíz I. N. y P. P. Saravia. 1989. Parque Nacional "Noel Kempff Mercado". Plan Operativo 1990-1995. Santa Cruz, Bolivia. $74 \mathrm{pp}$.

Sarmiento G. 1983. The savannas of tropical américa. En: Bourlière, F. (ed.). Ecosystems of the world 13. Tropical Savannas. Elsevier Scientific Publishing Company. 245288 pp.

Sarmiento G. y M. Monasterio. 1983. Life forms and phenology. En: Bourlière, F. (ed.). Ecosystems of the world 13. Tropical Savannas. Elsevier Scientific Publishing Company. 79-108 pp.

Smith R.L. 1980. Ecology and Field Biology. 3ra. ed. New York, U.S.A. 664-695 pp. 
Apéndice 1. Lista de plantas registradas en los dos sitios de estudio, indicando las formas de crecimiento

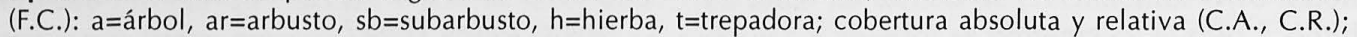
frecuencia absoluta y relativa (F.A., F.R.); y el número de espécimen (ESP.) colectado por Bonifacio Mostacedo.

\begin{tabular}{|c|c|c|c|c|c|c|c|c|c|c|}
\hline \multirow[t]{2}{*}{ NOMBRE CIENTÍFICO } & \multicolumn{5}{|c|}{ Sitio 1} & \multicolumn{4}{|c|}{ Sitio 2} & \multirow[b]{2}{*}{ Espécimen } \\
\hline & F.C. & $\mathrm{CA}$ & CR $(\%)$ & FA & FR $(\%)$ & $\mathrm{CA}$ & CR (\%) & $\mathrm{FA}$ & FR $(\%)$ & \\
\hline \multicolumn{11}{|l|}{ Adiantaceae } \\
\hline Adiantum sp.1 & h & & & & & 2 & 0.01 & 1 & 0.37 & 1268 \\
\hline Adiantum sp. 2 & h & & & & & 9 & 0.05 & 2 & 0.73 & 1349 \\
\hline \multicolumn{11}{|l|}{ Annonaceae } \\
\hline $\begin{array}{l}\text { Annona dioica A.St. Hil. } \\
\text { Duguetia furfuracea }\end{array}$ & ar & 11 & 0.09 & 1 & 0.29 & & & & & 1030 \\
\hline $\begin{array}{l}\text { (A. Saint-Hil.) Benth. \& H } \\
\text { Xylopia aff. aromatica }\end{array}$ & ar & 56 & 0.46 & 2 & 0.57 & & & & & 1230 \\
\hline $\begin{array}{l}\text { (Lam.) Mart. } \\
\text { Apocynaceae }\end{array}$ & ar & & & & & 90 & 0.54 & 1 & 0.37 & 1376 \\
\hline Aspidosperma? sp. & $\mathrm{sb}$ & & & & & 50 & 0.30 & 1 & 0.37 & 1624 \\
\hline $\begin{array}{l}\text { Hancornia speciosa Gomez } \\
\text { Himatanthus obovatus }\end{array}$ & $\mathrm{a}$ & & & & & 305 & 1.84 & 2 & 0.73 & 1311 \\
\hline (Muell. Arg.) Wood. & ar & & & & & 3 & 0.01 & 1 & 0.37 & 1307 \\
\hline Himatanthus sp. & ar & & & & & 1 & 0.01 & 1 & 0.37 & 1352 \\
\hline $\begin{array}{l}\text { Mandevilla aff. angustifolia } \\
\text { (Malme) Woods }\end{array}$ & $\mathrm{h}$ & & & & & 4 & 0.02 & 2 & 0.73 & 1355 \\
\hline $\begin{array}{l}\text { Apocynaceae sp. } \\
\text { Araliaceae }\end{array}$ & $\mathrm{h}$ & & & & & 7 & 0.04 & 1 & 0.37 & 1323 \\
\hline Didymopanax distractiflorus & & & & & & & & & & \\
\hline $\begin{array}{l}\text { Harms } \\
\text { Bignoniaceae }\end{array}$ & ar & & & & & 41 & 0.25 & 2 & 0.73 & 1336 \\
\hline $\begin{array}{l}\text { Tabebuia aff. roseo-alba } \\
\text { (Ridley) Sandw. }\end{array}$ & a & 200 & 1.63 & 1 & 0.29 & & & & & 1178 \\
\hline $\begin{array}{l}\text { Tabebuia aurea } \\
\text { (A. Silva Manso) }\end{array}$ & & & & & & & & & & \\
\hline $\begin{array}{l}\text { B.\&H. ex. S. M } \\
\text { Bombacaceae }\end{array}$ & a & 10 & 0.08 & 1 & 0.29 & & & & & - \\
\hline $\begin{array}{l}\text { Eriotheca cf. gracilipes } \\
\text { (Schumann) Robyns }\end{array}$ & a & 310 & 2.52 & 2 & 0.57 & & & & & 1224 \\
\hline Eriotheca sp. & a & 10 & 0.08 & 1 & 0.29 & 168 & 1.01 & 2 & 0.73 & 1581,1273 \\
\hline Pseudobombax sp. & a & 14 & 0.11 & 1 & 0.29 & 198 & 1.20 & 3 & 1.10 & 1163,1348 \\
\hline $\begin{array}{l}\text { Pseudobombax longiflorum } \\
\text { (C. Martius\&Zucc.) } \\
\text { Boraginaceae }\end{array}$ & a & 30 & 0.24 & 2 & 0.57 & & & & & 1112 \\
\hline $\begin{array}{l}\text { Cordia buddleoides Rusby } \\
\text { Caryocariaceae }\end{array}$ & $\mathrm{h}$ & 5 & 0.04 & 1 & 0.29 & & & & & 1221 \\
\hline $\begin{array}{l}\text { Caryocar brasiliense Cambess } \\
\text { Celastraceae }\end{array}$ & a & 165 & 1.34 & 3 & 0.86 & 220 & 1.33 & 3 & 1.10 & \\
\hline $\begin{array}{l}\text { Maytenus sp. } \\
\text { Chrysobalanaceae }\end{array}$ & a & 400 & 3.26 & 3 & 0.86 & 70 & 0.42 & 2 & 0.73 & 1032,1280 \\
\hline $\begin{array}{l}\text { Chrysobalanaceae } \\
\text { Licania gardneriana }\end{array}$ & & & & & & & & & & \\
\hline $\begin{array}{l}\text { (Hook. f) Fritsch } \\
\text { Combretaceae }\end{array}$ & a & & & & & 53 & 0.32 & 2 & 0.73 & 1371 \\
\hline Buchenavia aff. tomentosa & & & & & & & & & & \\
\hline Eichler & ar & & & & & 43 & 0.26 & 1 & 0.37 & 1324 \\
\hline Compositae & & & & & & & & & & \\
\hline Compositae 1 & $\mathrm{~h}$ & 3 & 0.02 & 3 & 0.86 & & & & & 1148 \\
\hline Compositae 2 & $\mathrm{~h}$ & 3 & 0.02 & 1 & 0.27 & & & & & 1240 \\
\hline Compositae 3 & $\mathrm{~h}$ & & & & & 5 & 0.03 & 2 & 0.73 & 1325 \\
\hline Compositae 4 & h & & & & & 14 & 0.08 & 1 & 0.37 & 1618 \\
\hline
\end{tabular}




\begin{tabular}{|c|c|c|c|c|c|c|c|c|c|c|}
\hline \multirow[t]{2}{*}{ NOMBRE CIENTÍFICO } & \multicolumn{5}{|c|}{ Sitio 1} & \multicolumn{4}{|c|}{ Sitio 2} & \multirow[b]{2}{*}{ Espécimen } \\
\hline & F.C. & $\mathrm{CA}$ & CR (\%) & FA & FR $(\%)$ & $\mathrm{CA}$ & CR $(\%)$ & FA & FR $(\%)$ & \\
\hline \multicolumn{11}{|l|}{ Connaraceae } \\
\hline \multicolumn{11}{|l|}{ Connarus suberosus } \\
\hline Planchon & $\mathrm{a}$ & 41 & 0.33 & 2 & 0.57 & & & & & 1115 \\
\hline \multicolumn{11}{|l|}{ Cycadaceae } \\
\hline \multicolumn{11}{|l|}{ Zamia boliviensis } \\
\hline (Brongn.) A. DC. & $\mathrm{sb}$ & 75 & 0.61 & 5 & 1.44 & & & & & 1094 \\
\hline \multicolumn{11}{|l|}{ Cyperaceae } \\
\hline Bulbostylis sp. 1 & $g r$ & 24 & 0.19 & 4 & 1.15 & 1 & 0.01 & 1 & 0.37 & 1012,1012 \\
\hline Bulbostylis sp. 2 & $\mathrm{gr}$ & 5 & 0.04 & 1 & 0.28 & & & & & 1606 \\
\hline Frimbistilis sp. & $\mathrm{gr}$ & & & & 年 & 1 & 0.01 & 1 & 0.37 & 1308 \\
\hline Rhynchospora sp. & $\mathrm{gr}$ & & & & & 2 & 0.01 & 1 & 0.37 & 1623 \\
\hline Cyperaceae sp. 1 & $\mathrm{gr}$ & & & & & 1 & 0.01 & 1 & 0.37 & 1622 \\
\hline \multicolumn{11}{|l|}{ Dilleniaceae } \\
\hline \multicolumn{11}{|l|}{ Davilla grandiflora } \\
\hline A.St. Hil.\&Tusl. & ar & 465 & 3.78 & 9 & 2.59 & 141 & 0.85 & 8 & 2.93 & 1021,1361 \\
\hline \multicolumn{11}{|l|}{ Dioscoriaceae } \\
\hline \multicolumn{11}{|l|}{ Dioscorea amaranthoides } \\
\hline J. S. Presl & $\mathrm{t}$ & 7 & 0.06 & 1 & 0.29 & 2 & 0.01 & 1 & 0.37 & 1225,1356 \\
\hline \multicolumn{11}{|l|}{ Ebenaceae } \\
\hline Dyospiros hispada DC. A. & ar & & & & & 42 & 0.25 & 1 & 0.37 & 1330 \\
\hline Dyospiros sp. & $\mathrm{h}$ & & & & & 2 & 0.01 & 1 & 0.37 & 1284 \\
\hline \multicolumn{11}{|l|}{ Erythroxylaceae } \\
\hline $\begin{array}{l}\text { Erythroxylum cf. } \\
\text { angifugum Martius }\end{array}$ & \multicolumn{10}{|c|}{ Erythroxylum cf. } \\
\hline Erythroxylum suberosum & & & & & & & & & & \\
\hline A. St. Hil. & $\mathrm{sb}$ & 13 & 0.10 & 3 & 0.86 & 42 & 0.25 & 4 & 1.47 & 1121,1275 \\
\hline Erythroxylum tortuosum & & & & & & & & & & \\
\hline Martius & ar & & & & & 3 & 0.02 & 1 & 0.37 & 1305 \\
\hline Erythroxylum sp. & $\mathrm{sb}$ & & & & & 6 & 0.04 & 1 & 0.37 & 1277 \\
\hline Euphorbiaceae & & & & & & & & & & \\
\hline Croton ackermannianus & & & & & & & & & & \\
\hline (Muell. Arg.) Webster & h & 2 & 0.02 & 1 & 0.29 & & & & & 1182 \\
\hline Croton sp. & $\mathrm{h}$ & & & & & 1 & 0.01 & 1 & 0.37 & 1310 \\
\hline Euphorbia coccorum Martius & $\mathrm{h}$ & 3 & 0.02 & 1 & 0.29 & & & & & 1090 \\
\hline Hyeronima sp. & ar & 61 & 0.49 & 3 & 0.86 & & & & & 1223 \\
\hline Manihot caurulescens Pohl. & h & & & & & 21 & 0.13 & 5 & 1.83 & 1285 \\
\hline Sebastiana corniculata & & & & & & & & & & \\
\hline (M. Vahl) Muell. Arg. & h & 1 & 0.01 & 1 & 0.29 & & & & & 1598 \\
\hline Sebastiana glandulosa & & & & & & & & & & \\
\hline (Martius) Pax & $\mathrm{h}$ & 3 & 0.03 & 2 & 0.58 & & & & & 1079,1227 \\
\hline Euphorbiaceae sp.1 & $\mathrm{h}$ & 10 & 0.08 & 1 & 0.29 & & & & & 1233 \\
\hline Flacourtiaceae & & & & & & & & & & \\
\hline Casearia javitensis Kunth & ar & & & & & 60 & 0.36 & 4 & 1.47 & 1320 \\
\hline Casearia sylvestris Kunth & $\mathrm{ar}$ & 102 & 0.83 & 4 & 1.15 & 5 & 0.03 & 1 & 0.37 & 1104,1337 \\
\hline Gramineae & & & & & & & & & & \\
\hline Actinocladum verticillatum & & & & & & & & & & \\
\hline (Nees) McClure ex & ar & & & & & 30 & 0.18 & 1 & 0.37 & 1363 \\
\hline Aristida riparia Trin. & gr & & & & & 7 & 0.04 & 1 & 0.37 & \\
\hline Axonopus aff. marginatus & & & & & & & & & & \\
\hline (Trin.) Chase & $\mathrm{gr}$ & 849 & 6.91 & 12 & 3.45 & & & & & 1004 \\
\hline $\begin{array}{l}\text { Axonopus aureus P. Beauv. } \\
\text { Digitaria dioica }\end{array}$ & $\mathrm{gr}$ & 51 & 0.41 & 1 & 0.29 & 3 & 0.02 & 1 & 0.37 & 1629 \\
\hline Killeen \& Rugolo & gr & 357 & 2.91 & 10 & 2.87 & 247 & 1.49 & 9 & 3.30 & 990,1384 \\
\hline Elyonurus muticus (Sprengel) & & & & & & & & & & \\
\hline Kuntze & $\mathrm{gr}$ & 2718 & 22.12 & 11 & 3.16 & & & & & 1151 \\
\hline Eragrostis sp. & $\mathrm{gr}$ & & & & & 6 & 0.04 & 1 & 0.37 & 1300 \\
\hline
\end{tabular}


Sitio 1

F.C. $\quad \mathrm{CA} \quad \mathrm{CR}(\%) \quad \mathrm{FA} \quad \mathrm{FR}(\%)$

Sitio 2
Ichnanthus procurrens

(Nees ex Trin.) Swallen

Mesosetum cayennense Steudel

Panicum aff. cyanescens

Nees ex Trin.

Panicum? sp.

Paspalum gemmiflorum Steudel

Paspalum pectinatum

Nees ex Trin.

Paspalum sp.

Schyzachirium sanguineum

(Retz.)Alston

Sporobolus cubensis A. Hitchc.

Thrasya petrosa (Trin.)Chase

Trachypogon plumosus

(Humb. \& Bonpl. ex Will

Gramineae sp. 1

Gramineae sp. 2

Gramineae sp. 3

Gramineae sp.4

Gramineae sp. 5

Gramineae sp.6

Gramineae sp.7

Gramineae sp. 8

Gramineae sp. 9

Gramineae sp. 10

Gramineae sp. 11

Guttiferae

Caraipa aff. densifolia Martius

Kielmeyera coriacea Martius

Kielmeyera rubriflora Cambess

Icacinaceae

Emmotum nitens Miers

$\mathrm{gr}$
$\mathrm{gr}$
$\mathrm{gr}$
$\mathrm{gr}$
$\mathrm{gr}$
$\mathrm{gr}$
$\mathrm{gr}$
$\mathrm{gr}$
$\mathrm{gr}$
$\mathrm{gr}$
$\mathrm{gr}$
$\mathrm{gr}$

$\mathrm{gr}$

123

10

2.87

11

0.07

3

1.10

$\begin{array}{lllll}\text { gr } & 9 & 0.07 & 1 & 0.29\end{array}$

$\begin{array}{lllll}\text { gr } & 7 & 0.05 & 3 & 0.86\end{array}$

$\begin{array}{lllll}\text { gr } & 71 & 0.58 & 5 & 1.44\end{array}$

$\begin{array}{lllll}\text { gr } & 1312 & 10.68 & 11 & 3.16\end{array}$

$\begin{array}{lllll}\text { gr } & 19 & 0.15 & 2 & 0.58\end{array}$

$\begin{array}{lllll}\text { gr } & 10 & 0.08 & 1 & 0.29\end{array}$

$\mathrm{gr}$

gr

4

$\begin{array}{llll}4 & 0.03 & 1 & 0.29\end{array}$

$\mathrm{gr}$
$\mathrm{gr}$

gr

gr Iridaceae

Sisyrinchum vaginatum Sprengel gr Labiatae

Hyptis sp.1

Hyptis sp. 2

Leguminosae

Aeschynomene oroboide Kuntz

Bahuinia rufa (Bong.) Steudel

Bauhinia glabra Jacq.

Bowdichia virgiliodes Kunth

Chamaecrista diphylla

$$
\text { (L.) Greene }
$$

Chamaecrista desvauxii

$$
\text { (Colladon) Killip }
$$

Chamaecrista sp.

Clitoria sp.1

Clitoria sp. 2

Crotalaria? sp.

Diptychandra aurantiaca Tul.

Entada? sp.

Indigofera lespedezioides Kunth

Mimosa sp.

Ormosia sp.

Plathymenia reticulata Benth. a
ar
ar

a

gr

h

$\mathrm{h}$

h

$\begin{array}{lllll}\text { sb } & 235 & 1.91 & 6 & 1.72\end{array}$

$\begin{array}{lrlll}\text { sb } & 20 & 0.16 & 2 & 0.57\end{array}$

h

h

h

h

t

h

h

ar

h

a

$\begin{array}{llll}11 & 0.09 & 2 & 0.57\end{array}$

$\begin{array}{llll}7 & 0.06 & 4 & 1.15\end{array}$

$\begin{array}{llll}90 & 0.73 & 1 & 0.29\end{array}$

$\begin{array}{llll}5 & 0.04 & 1 & 0.29\end{array}$

$\begin{array}{llll}156 & 1.27 & 2 & 0.58\end{array}$

$\begin{array}{rlrl}188 & 1.53 & 2 & 0.57 \\ 16 & 0.13 & 3 & 0.86 \\ 63 & 0.51 & 7 & 2.01 \\ & & & \\ & & & \\ 4 & 0.03 & 1 & 0.29 \\ 38 & 0.31 & 2 & 0.57 \\ 224 & 1.82 & 10 & 2.87 \\ 160 & 1.3 & 2 & 0.57 \\ & & & \\ & & & \\ 126 & 1.03 & 7 & 2.01 \\ 60 & 0.49 & 1 & 0.29 \\ 22 & 0.17 & 2 & 0.58\end{array}$

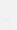

15

0.09

0.37

$\begin{array}{rrrrr}27 & 0.16 & 3 & 1.10 & 1342,1596 \\ 497 & 3.00 & 10 & 3.66 & 1014,1282 \\ & & & & 1584\end{array}$

1149

1194

$\begin{array}{rrrrr}23 & 0.14 & 1 & 0.37 & 1605,1631 \\ 457 & 2.76 & 7 & 2.56 & 1261 \\ 24 & 0.14 & 1 & 0.37 & 1018,1625\end{array}$

$\begin{array}{lllll}3737 & 22.56 & 11 & 4.03 & 1592,1260\end{array}$

$\begin{array}{rrrrr}5 & 0.03 & 1 & 0.37 & 1340\end{array}$

$\begin{array}{lllll}8 & 0.05 & 1 & 0.37 & 1152,1386\end{array}$

$\begin{array}{lllll}47 & 0.28 & 1 & 0.37 & 1580\end{array}$

$\begin{array}{llll}732 & & 1011\end{array}$

$\begin{array}{lllll}732 & 4.42 & 3 & 1.10 & 1370\end{array}$

$\begin{array}{lllll}409 & 2.47 & 9 & 3.30 & 1295\end{array}$

$\begin{array}{lllll}225 & 1.36 & 6 & 2.20 & 1611,1334\end{array}$

1157

1019

1589

$\begin{array}{lllll}1953 & 11.79 & 6 & 2.20 & 1322\end{array}$

$\begin{array}{lllll}3293 & 19.88 & 10 & 3.66 & 1025,1278\end{array}$

$\begin{array}{lllll}45 & 0.27 & 1 & 0.37 & 1095\end{array}$

$\begin{array}{lllll}31 & 0.19 & 2 & 0.73 & 996,1267\end{array}$

$\begin{array}{lllll}345 & 2.08 & 3 & 1.10 & 1345\end{array}$

1122

1009

1591

$\begin{array}{lllll}14 & 0.08 & 1 & 0.37 & 1315\end{array}$

1033

1102

$\begin{array}{lllll}220 & 1.33 & 3 & 1.10 & 1274\end{array}$

1198

1165

1241

1612

$\begin{array}{lllll}20 & 0.12 & 1 & 0.37 & 1180,1100\end{array}$

1351

1197

1330

1192

1626

1237

1173 


\begin{tabular}{|c|c|c|c|c|c|c|c|c|c|c|}
\hline \multirow[t]{2}{*}{ NOMBRE CIENTÍFICO } & \multicolumn{5}{|c|}{ Sitio 1} & \multicolumn{4}{|c|}{ Sitio 2} & \multirow[b]{2}{*}{ Espécimen } \\
\hline & F.C. & $\mathrm{CA}$ & CR $(\%)$ & FA & FR $(\%)$ & $\mathrm{CA}$ & CR (\%) & FA & FR $(\%)$ & \\
\hline \multicolumn{11}{|l|}{ Senna sylvestris (Vell.) } \\
\hline \multicolumn{11}{|l|}{ Stylosanthes parviflora } \\
\hline M. B. Ferr. \& Souza & h & & & & & 4 & 0.02 & 1 & 0.37 & 1299 \\
\hline Vigna firmula (Hassler) Mane & $\mathrm{h}$ & & & & & 13 & 0.08 & 1 & 0.37 & 1290 \\
\hline Leguminosae sp.1 & ar & & & & & 21 & 0.13 & 1 & 0.37 & 1332 \\
\hline Leguminosae sp.2 & $\mathrm{h}$ & 2 & 0.02 & 1. & 0.29 & & & & & 1222 \\
\hline Leguminosae sp.3 & ar & 15 & 0.12 & 1 & 0.29 & & & & & 1228 \\
\hline Leguminosae sp.4 & $\mathrm{a}$ & 20 & 0.16 & 1 & 0.29 & 80 & 0.48 & 1 & 0.37 & 1595,1332 \\
\hline \multicolumn{11}{|l|}{$\begin{array}{l}\text { Lythraceae } \\
\text { Cuphea aff. sperm }\end{array}$} \\
\hline A. St. Hil. & $\mathrm{h}$ & 6 & 0.05 & 2 & 0.58 & 4 & 0.02 & 1 & 0.37 & 1092,1312 \\
\hline Lafoensia vandelliana Koehne & a & 268 & 2.18 & 4 & 1.15 & 10 & 0.06 & 1 & 0.37 & 1147,1139 \\
\hline Lythraceae sp. 1 & $\mathrm{~h}$ & & & & & 4 & 0.02 & 2 & 0.73 & 1309 \\
\hline \multicolumn{11}{|l|}{ Malpighiaceae } \\
\hline Byrsonima coccolobifolia Kunth & a & 20 & 0.16 & 1 & 0.29 & & & & & 1218 \\
\hline Byrsonima crassifolia (L.) Kunth & ar & & & & & 180 & 1.09 & 2 & 0.73 & 1634 \\
\hline $\begin{array}{l}\text { Byrsonima fagifolia Nieden } \\
\text { Tetrapterys ambigua }\end{array}$ & a & 253 & 2.06 & 7 & 2.01 & & & & & 1179 \\
\hline (Adr. Juss) Nieden & $\mathrm{sb}$ & 101 & 0.82 & 5 & 1.44 & & & & & 1229 \\
\hline \multicolumn{11}{|l|}{ Malvaceae } \\
\hline Pavovia aff. boliviana Fryx. & $\mathrm{h}$ & 26 & 0.21 & 2 & 0.57 & & & & & 1597 \\
\hline Malvaceae sp.1 & h & 10 & 0.08 & 2 & 0.57 & & & & & 1084 \\
\hline \multicolumn{11}{|l|}{ Melastomataceae } \\
\hline \multicolumn{11}{|l|}{ Miconia aff. rufescens } \\
\hline (Aublet) DC. & $\mathrm{h}$ & 4 & 0.03 & 1 & 0.29 & & & & & 1236 \\
\hline Miconia albicans (Sw.) Triana & ar & & & & & 418 & 2.52 & 12 & 4.40 & 1281,1344 \\
\hline Miconia sp. & ar & & & & & 35 & 0.21 & 2 & 0.73 & 1286 \\
\hline \multicolumn{11}{|l|}{ Reincourtia aff. oblongifolia } \\
\hline (Baker) Kunth\&R & h & & & & & 73 & 0.44 & 5 & 1.83 & 1264 \\
\hline Tibouchina sp. & $\mathrm{h}$ & 5 & 0.04 & 2 & 0.57 & & & & & 1070 \\
\hline Melastomataceae sp.1 & h & & & & & 1 & 0.01 & 1 & 0.37 & 1303 \\
\hline \multicolumn{11}{|l|}{ Moraceae } \\
\hline $\begin{array}{l}\text { Brosimun gaudichaudii Trécul } \\
\text { Sorocea aff. guilleminiana }\end{array}$ & ar & 34 & 0.28 & 2 & 0.57 & & & & & 1026 \\
\hline Gaudin & h & 1 & 0.01 & 1 & 0.29 & & & & & 1081 \\
\hline \multirow{2}{*}{\multicolumn{11}{|c|}{$\begin{array}{l}\text { Myrsinaceae } \\
\text { Cybianthus penduliflorus }\end{array}$}} \\
\hline \multicolumn{10}{|l|}{ Cybianthus penduliflorus } & \\
\hline Martius & $\mathrm{sb}$ & & & & & 10 & 0.06 & 1 & 0.37 & 1288 \\
\hline \multicolumn{11}{|l|}{ Myrtaceae } \\
\hline Eugenia parviflora (L.) DC. & $\mathrm{sb}$ & 10 & 0.08 & 3 & 0.86 & 15 & 0.09 & 2 & 0.73 & 1195,1366 \\
\hline Eugenia punicifolia (Kunth) DC. & $\mathrm{sb}$ & 24 & 0.19 & 2 & 0.57 & & & & & 1128 \\
\hline Myrcia aff. regnelliana O. Berg. & ar & 25 & 0.20 & 1 & 0.29 & 118 & 0.71 & 3 & 1.10 & 1607,1287 \\
\hline Myrcia guianensis (Aublet) DC. & $\mathrm{sb}$ & & & & & 80 & 0.48 & 1 & 0.37 & 1338 \\
\hline Myrcia sp. 1 & $\mathrm{sb}$ & 82 & 0.67 & 1 & 0.29 & 38 & 0.23 & 8 & 2.93 & 1134,1294 \\
\hline Myrcia sp. 2 & $\mathrm{sb}$ & 26 & 0.21 & 4 & 1.15 & & & & & 1053 \\
\hline Myrcia sp. 3 & $\mathrm{sb}$ & 25 & 0.15 & 1 & 0.37 & & & & & 1326 \\
\hline Myrcia sp. 4 & ar & & & & & 1 & 0.01 & 1 & 0.37 & 1296 \\
\hline Psidium salutare & & & & & & & & & & \\
\hline (Kunth) O. Berg & $\mathrm{sb}$ & 330 & 2.69 & 10 & 2.87 & & & & & \\
\hline Psidium sp. & $\mathrm{sb}$ & 38 & 0.31 & 2 & 0.57 & & & & & 1099 \\
\hline Nyctaginaceae & & & & & & & & & & \\
\hline Neea cf. ovalifolia & & & & & & & & & & \\
\hline Spruce ex J.A. Schmidt & $\mathrm{sb}$ & 5 & 0.04 & 1 & 0.29 & & & & & 1193 \\
\hline Nyctaginaceae sp. 1 & $\mathrm{sb}$ & 47 & 0.38 & 2 & 0.57 & & & & & 1085 \\
\hline Ochnaceae & & & & & & & & & & \\
\hline Ouratea spectabilis & & & & & & & & & & \\
\hline (Martius) Engl. & $\mathrm{sb}$ & 240 & 1.96 & 12 & 3.44 & 30 & 0.18 & 2 & 0.37 & 1052,1638 \\
\hline
\end{tabular}




\begin{tabular}{lllllllllllll}
\hline F.C. & CA & CR (\%) & FA & FR (\%) & CA & CR (\%) & FA & FR (\%) & Espécimen
\end{tabular}

Ouratea sp.

$\mathrm{sb}$

Opiliaceae

Agonandra brasiliensis Miers ex Benth.

Polygalaceae

Polygala cf. timoutoides Chodat

Polygala sp.

Proteaceae

Roupala montana Aublet

Rubiaceae

Alibertia cf. steinbachii Standley ar Alibertia edulis (Rich.)

$$
\text { A. Rich. ex. DC.) }
$$

Borreria sp.

Faramea aff. sessifolia

$$
\text { (Kunth) DC. }
$$

Palicourea coriacea (Cham.)

Schumann

Palicourea sp.

Rudgea aff. cornifolia

(Humb.\&Bonpl. ex Roe

Sabicea sp.

Tocoyena foetida Poeppig

Rubiaceae sp. 1

Sapindaceae

Matayba guianensis Aublet

Serjania reticulata Cambess

Sapotaceae

Pouteria ramiflora (Mart.) Radlk.

Smilacaceae

Smilax aff. rufescens Griseb.

Smilax sp.

Sterculiaceae

Helicteres sp.

Styracaceae

Styrax sp.

Tiliaceae

Corchorus sp.

Verbenaceae

Hyptis aff. parkeri Benth

Lippia velutina Schau

Lippia sp. 1

Lippia sp. 2

Lippia sp. 3

Verbenaceae sp.1-

Verbenaceae sp.2

Verbenaceae sp.3

Vochysiaceae

Callistene fasciculata

Qualea grandiflora Mart.

Salvertia convallariodora St. Hil. Vochysia sp.

Vochysiaceae sp.1

Xyridaceae

Xyridaceae sp. 1

Indeterminadas

Indet. 1

h

a

ar

h

sb

(

$\mathrm{a}$
$\mathrm{h}$
$\mathrm{ar}$

ar

$\mathrm{sb}$

$\mathrm{t}$

a

t

t

h

ar

h

h

h
44

0.26

$\begin{array}{ll}6 & 2.20\end{array}$

1265

1170

$\begin{array}{lllll}\text { a } & 97 & 0.79 & 2 & 0.57\end{array}$

$0.02 \quad 1 \quad 0.37$

1369

$\begin{array}{llll}5 & 0.04 & 1 & 0.29\end{array}$

$\begin{array}{lllll}233 & 1.90 & 5 & 1.44 & 352\end{array}$

2.12

2.93

988,1321

599

4.88

11

3.16

10

0.06

0.37

1339

20

0.12

0.37

1318

998

6

0.04

0.37

1389

1

1
10
6

$\begin{array}{lll}0.01 & 1 & 0.29\end{array}$

0.58

$\begin{array}{lll}0.05 & 1 & 0.29\end{array}$

3
5

0.02

0.37

1289

5

0.03

0.37

1329

1154

1129,1216

1111

$\begin{array}{lllll}20 & 0.12 & 1 & 0.37 & 1635\end{array}$

$\begin{array}{lllll}80 & 0.48 & 3 & 1.10 & 1357\end{array}$

1234

$\begin{array}{llll}9 & 0.07 & 1 & 0.29\end{array}$

180

1.096

$2.20 \quad 1023,1270$

68

0.41

7

2.56

1292

1232

1027

$\begin{array}{llll}43 & 0.35 & 5 & 1.44\end{array}$

6

0.04

0.37

1627

1615

h

$\begin{array}{lllll}\text { h } & 3 & 0.02 & 1 & 0.29\end{array}$

$\begin{array}{lllll}\text { h } & 12 & 0.02 & 1 & 0.29\end{array}$

h

$\begin{array}{llll}2 & 0.02 & 1 & 0.29\end{array}$

$\begin{array}{llll}4 & 0.03 & 1 & 0.29\end{array}$

$\begin{array}{llll}4 & 0.03 & 1 & 0.29\end{array}$

4

0.02

0.37

1353

1177

1167

$\begin{array}{lllll}5 & 0.03 & 1 & 0.37 & 1388\end{array}$

1024

1119

1162

10

0.06

0.37

1317

$\begin{array}{lllll}\text { a } & 25 & 0.20 & 1 & 0.29\end{array}$

20

0.12

0.37

1181

1630

$\begin{array}{llllll}\text { a } & 38 & 0.31 & 1 & 0.29 & 50\end{array}$

$\begin{array}{lllll}\text { ar } & 35 & 0.28 & 1 & 0.29\end{array}$

$0.30 \quad 1$

0.37

1017,1637

1175

1191

1588

1186 
Estructura y composición florística del Cerrado en el Parque Nacional Noel Kempff Mercado, Santa Cruz, Bolivia

\begin{tabular}{|c|c|c|c|c|c|c|c|c|c|c|}
\hline \multirow[t]{2}{*}{ NOMBRE CIENTÍFICO } & \multicolumn{5}{|c|}{ Sitio 1} & \multicolumn{4}{|c|}{ Sitio 2} & \multirow[b]{2}{*}{ Espécimen } \\
\hline & F.C. & $\mathrm{CA}$ & CR (\%) & $\mathrm{FA}$ & FR (\%) & $\mathrm{CA}$ & CR (\%) & FA & FR (\%) & \\
\hline Indet. 2 & $\mathrm{~h}$ & 6 & 0.05 & 1 & 0.29 & & & & & 1242 \\
\hline Indet. 3 & $\mathrm{~h}$ & & & & & 3 & 0.02 & 1 & 0.37 & 1277 \\
\hline Indet. 4 & ar & & & & & 16 & 0.10 & 3 & 1.10 & 1304 \\
\hline Indet. 5 & $\mathrm{~h}$ & & & & & 2 & 0.01 & 1 & 0.37 & 1347 \\
\hline Indet. 6 & h & & & & & 7 & 0.04 & 1 & 0.37 & 1354 \\
\hline Indet. 7 & $\mathrm{~h}$ & & & & & 3 & 0.02 & 1 & 0.37 & 1365 \\
\hline Indet. 8 & $\mathrm{sb}$ & & & . & & 21 & 0.13 & 1 & 0.37 & 1368 \\
\hline Indet. 9 & $\mathrm{~h}$ & & & & & 4 & 0.02 & 1 & 0.37 & 1620 \\
\hline
\end{tabular}

